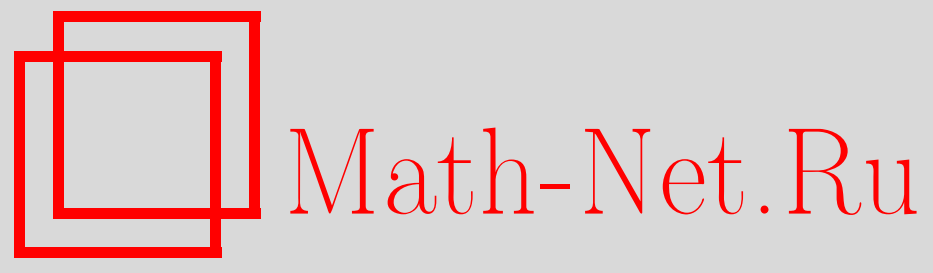

А. Ф. Заусаев, М. А. Романюк, Сравнение различных математических моделей на примере решения уравнений движения больших планет и Луны, Вестн. Сам. гос. техн. ун-та. Сер. Физ.-мат. науки, 2019, номер 1, 152-185

DOI: https://doi.org/10.14498/vsgtu1663

Использование Общероссийского математического портала MathNet.Ru подразумевает, что вы прочитали и согласны с пользовательским соглашением

http: //www . mathnet.ru/rus/agreement

Параметры загрузки:

IP: 54.237 .59 .107

26 апреля 2023 г., 14:28:28

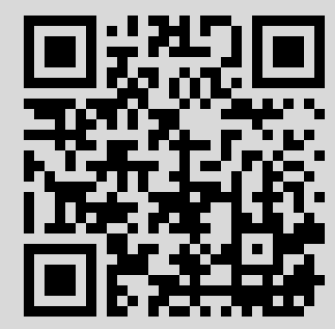


Вестн. Сам. гос. техн. ун-та. Сер. Физ.-мат. науки. 2019. Т. 23, № 1. С. $152-185$ ISSN: 2310-7081 (online), 1991-8615 (print)

УДК 521.182

\title{
Сравнение различных математических моделей на примере решения уравнений движения больших планет и Луны
}

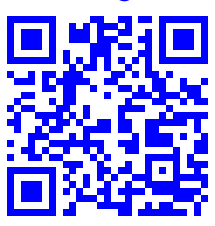

\section{А. Ф. Заусаев, М. А. Романюк}

Самарский государственный технический университет, Россия, 443100, Самара, ул. Молодогвардейская, 244.

\begin{abstract}
Аннотация
Проведено исследование точности решения различных дифференциальных уравнений, описывающих движение больших планет, Луны и Солнца. На интервале времени с 31 года до нашей эры по 3969 год нашей эры проведено численное интегрирование ньютоновых, релятивистских дифференциальных уравнений и уравнений, полученных на основе взаимодействия окружающего пространства с движущимися материальными телами. Выявлена область применимости рассмотренных дифференциальных уравнений для исследуемых объектов. Путем сравнения координат Луны, найденных с помощью решения различных дифференциальных уравнений и банка данных DE405, показано, что наибольшая точность в элементах орбит больших планет и Луны достигается путем решения дифференциальных уравнений, полученных на основе взаимодействия окружающего пространства с движущимися материальными телами. Решение релятивистских уравнений обеспечивает высокую точность элементов орбит для Меркурия и внешних планет на всем интервале интегрирования. Однако для остальных внутренних планет и Луны точность элементов орбит, полученных с помощью решения релятивистских уравнений, сопоставима с точностью, полученной путем решения ньютоновых уравнений. Полагается, что использование гармонической системы координат является обоснованным лишь для Меркурия с точки зрения скорости векового смещения долготы его перигелия, однако для других внутренних планет (Венеры, Земли+Луны и Марса) скорости вековых смещений долгот перигелиев оказываются завышенными. Показано, что решение дифференциальных уравнений, полученных на основе взаимодействия окружающего пространства с движущимися материальными телами, обеспечивает более высокую точность по сравнению с решениями ньютоновых и релятивистских уравнений получения
\end{abstract}

\section{Научная статья}

ə () (7) Контент публикуется на условиях лицензии Creative Commons Attribution 4.0 International (https://creativecommons.org/licenses/by/4.0/deed.ru)

\section{Образец для цитирования}

З ау са е в А. Ф., Р ом ан юк М. А. Сравнение различных математических моделей на примере решения уравнений движения больших планет и Луны // Bестн. Сам. гос. техн. ун-та. Сер. Физ.-мат. науки, 2019. Т. 23, № 1. С. 152-185. doi: 10.14498/vsgtu1663.

\section{Сведения об авторах}

Анатолий Федорович Заусаев (1) https://orcid.org/0000-0002-5035-9615 доктор физико-математических наук; профессор; каф. прикладной математики и информатики; e-mail: zausaev_af@mail.ru

Мария Анатольевна Романюк (1) https://orcid.org/0000-0003-0796-2061 кандидат технических наук; доцент; каф. прикладной математики и информатики; e-mail: zausmasha@mail.ru 
элементов орбит для всех рассматриваемых объектов на исследуемом интервале времени.

Ключевые слова: элементы орбит, численное интегрирование, дифференциальные уравнения движения.

Получение: 6 декабря 2018 г. / Исправление: 27 февраля 2019 г. / Принятие: 4 марта 2019 г. / Публикация онлайн: 15 марта 2019 г.

Целью данной работы является сравнение различных математических моделей, описывающих движение небесных тел в Солнечной системе. K таким моделям относятся: дифференциальные уравнения движения в ньютоновой форме, релятивистские уравнения движения и уравнения, основанные на взаимодействии окружающего пространства с движущимися материальными телами.

Дифференциальные уравнения движения в ньютоновой форме в задаче $n$ тел в прямоугольных координатах с началом в центре масс всей системы $n$ материальных точек имеют следующий вид $[1,2]$ :

$$
\left\{\begin{array}{l}
\frac{d^{2} X}{d t^{2}}=\sum_{i} k^{2} m_{i}\left(\frac{X_{i}-X}{\Delta_{i}^{3}}\right), \\
\frac{d^{2} Y}{d t^{2}}=\sum_{i} k^{2} m_{i}\left(\frac{Y_{i}-Y}{\Delta_{i}^{3}}\right), \\
\frac{d^{2} Z}{d t^{2}}=\sum_{i} k^{2} m_{i}\left(\frac{Z_{i}-Z}{\Delta_{i}^{3}}\right),
\end{array}\right.
$$

где $\Delta_{i}^{2}=\left(X_{i}-X\right)^{2}+\left(Y_{i}-Y\right)^{2}+\left(Z_{i}-Z\right)^{2} ; X, Y, Z$ - барицентрические координаты возмущаемого тела, а $m_{i}, X_{i}, Y_{i}, Z_{i}$ - массы и барицентрические координаты возмущающих тел.

Дифференциальные уравнения движения в барицентрической системе координат с учетом релятивистских членов представляются в виде [3]

$$
\begin{aligned}
\ddot{r}_{i}=\sum_{j \neq i} \frac{\mu_{j}\left(r_{j}-r_{i}\right)}{r_{i j}^{3}}\left\{1-\frac{2(\beta+\gamma)}{c^{2}} \sum_{k \neq i} \frac{\mu_{k}}{r_{i k}}-\frac{2 \beta-1}{c^{2}} \sum_{k \neq j} \frac{\mu_{k}}{r_{j k}}+\gamma\left(\frac{v_{i}}{c}\right)^{2}+\right. \\
\left.+(1+\gamma)\left(\frac{v_{j}}{c}\right)^{2}-\frac{2(1+\gamma)}{c^{2}} \dot{r}_{i} \dot{r}_{j}-\frac{3}{2 c^{2}}\left[\frac{\left(r_{i}-r_{j}\right) \dot{r}_{i}}{r_{i j}}\right]^{2}+\frac{1}{2 c^{2}}\left(r_{j}-r_{i}\right) \ddot{r}_{j}\right\}+ \\
+\frac{1}{c^{2}} \sum_{j \neq i} \frac{\mu_{j}}{r_{i j}^{3}}\left(r_{i}-r_{j}\right)\left[(2+2 \gamma) \dot{r}_{i}-(1+2 \gamma) \dot{r}_{j}\right]\left(\dot{r}_{i}-\dot{r}_{j}\right)+ \\
+\frac{3+4 \gamma}{2 c^{2}} \sum_{j \neq i} \frac{\mu_{j} \ddot{r}_{j}}{r_{i j}}+\sum_{m=1}^{n} \frac{\mu_{m}\left(r_{m}-r_{i}\right)}{r_{i m}^{3}}
\end{aligned}
$$

где $r_{i}, \dot{r}_{i}, \ddot{r}_{i}$ - координаты, скорости, ускорения в барицентрической системе координат $i$-того тела; $\mu_{j}=k^{2} m_{j}, k^{2}$ - гравитационная постоянная, $m_{j}-$ масса $j$-того тела; $r_{i j}=\left|r_{j}-r_{i}\right|$; релятивистские параметры $\beta$ и $\gamma$ в заданной 
системе координат принимают следующие значения: $\beta=\gamma=1, v_{i}=\left|\dot{r}_{i}\right| ; c-$ скорость света.

При вычислении координат Луны наряду с гравитационными и релятивистскими эффектами учитывается влияние несферичности фигур Земли и Луны в математической модели. Дифференциальные уравнения для учета ускорения Луны в геоцентрической системе координат ( $\eta \zeta)$ имеют вид [3]

$$
\begin{aligned}
& {\left[\begin{array}{c}
\ddot{\xi} \\
\ddot{\eta} \\
\ddot{\zeta}
\end{array}\right]=-\frac{\mu}{r^{2}}\left\{\sum_{n=1}^{n_{1}} J_{n}\left(\frac{a}{r}\right)^{n}\left[\begin{array}{c}
(n+1) P_{n}(\sin \varphi) \\
0 \\
-\cos \theta P_{n}^{\prime}(\sin \varphi)
\end{array}\right]+\right.} \\
& \left.+\sum_{n=1}^{n_{2}}\left(\frac{a}{r}\right)^{n} \sum_{m=1}^{n}\left[\begin{array}{c}
-(n+1) P_{n}^{m}(\sin \varphi)\left[C_{n m} \cos m \lambda+S_{n m} \sin m \lambda\right] \\
m \sec \varphi P_{n}^{m}(\sin \varphi)\left[-C_{n m} \sin m \lambda+S_{n m} \cos m \lambda\right] \\
\cos \varphi P_{n}^{\prime m}(\sin \varphi)\left[C_{n m} \cos m \lambda+S_{n m} \sin m \lambda\right]
\end{array}\right]\right\},
\end{aligned}
$$

где ось $\xi$ направлена из начала координат в центр Луны; ось $\eta$ перпендикулярна оси $\xi$ и направлена на восток; ось $\zeta$ перпендикулярна плоскости $\xi \eta$ и направлена на север; $\mu$ - гравитационная постоянная, умноженная на массу Земли; $r$ - расстояние между центрами масс двух тел; $n_{1}$ и $n_{2}$ - максимальные степени зональных и тессеральных гармоник несферичных тел соответственно; $P_{n}(\sin \varphi)$ - полином Лежандра степени $n ; P_{n}^{m}(\sin \varphi)$ - присоединенный полином Лежандра степени $n$ и порядка $m ; J_{n}$ - зональные гармоники от несферичности тела; $C_{n m}, S_{n m}$ - коэффициенты тессеральных гармоник; $\varphi$ - широта притягиваемого тела в фиксированной системе координат $\xi \eta \zeta ;$ $\lambda$ - восточная долгота притягиваемого тела.

Дифференциальные уравнения движения, основанные на взаимодействии окружающего пространства с движущимися материальными телами в барицентрической системе координат, имеют следующий вид [4-7]:

$$
\left\{\begin{array}{l}
\frac{d^{2} X}{d t^{2}}=\sum_{i}\left(\frac{X_{i}-X}{\Delta_{i}}\right) \frac{3 a_{0 i} r_{0 i}^{2}}{\Delta_{i}^{2}+\Delta_{i} \sqrt[3]{\Delta_{i}^{3}-r_{0 i}^{3}}+\sqrt[3]{\left(\Delta_{i}^{3}-r_{0 i}^{3}\right)^{2}}} \\
\frac{d^{2} Y}{d t^{2}}=\sum_{i}\left(\frac{Y_{i}-Y}{\Delta_{i}}\right) \frac{3 a_{0 i} r_{0 i}^{2}}{\Delta_{i}^{2}+\Delta_{i} \sqrt[3]{\Delta_{i}^{3}-r_{0 i}^{3}}+\sqrt[3]{\left(\Delta_{i}^{3}-r_{0 i}^{3}\right)^{2}}} \\
\frac{d^{2} Z}{d t^{2}}=\sum_{i}\left(\frac{Z_{i}-Z}{\Delta_{i}}\right) \frac{3 a_{0 i} r_{0 i}^{2}}{\Delta_{i}^{2}+\Delta_{i} \sqrt[3]{\Delta_{i}^{3}-r_{0 i}^{3}}+\sqrt[3]{\left(\Delta_{i}^{3}-r_{0 i}^{3}\right)^{2}}}
\end{array}\right.
$$

где $\Delta_{i}$ - расстояние от начала координат до материального объекта; $r_{0 i}-$ эффективный радиус $i$-того тела; $a_{0 i}$ - соответствующее ускорение для $i$-того тела на расстоянии $r_{0 i}$ от центра массы; $X, Y, Z$ - барицентрические координаты возмущаемого тела; $X_{i}, Y_{i}, Z_{i}$ - барицентрические координаты возмущающих тел.

В настоящее время разработан ряд высокоточных численных теорий движения больших планет [8-17]. Наиболее известной из них является численная теория [3], созданная сотрудниками НАCA Ньюхалом (X. X. Newhall), Стендиншем (Е. M. Standish), Вильямсом (J. G. Williams). Ими создан банк данных координат больших планет, Луны и Солнца - DE405 на интервале 
времени с 2305424.5 J.D. (1599 Dec 9) по 2525008.5 J.D. (2201 Feb 20). Koopдинаты планет в банке данных хранятся в форме коэффициентов полиномов Чебышева, которые обеспечивают достаточно плотную форму записи на диске. Координаты и скорости внутренних планет, полученные с помощью банка данных DE405, согласованы с радиолокационными наблюдениями, а все планеты согласованы с оптическими наблюдениями.

Под эффективностью математической модели в дальнейшем понимается быстродействие и точность результатов решения, полученных на основе использования данной модели.

Проверка эффективности математических моделей, описываемых дифференциальными уравнениями (1)-(4), проводилась путем сравнения результатов вычисления, полученных на их основе, с банком данных DE405.

С целью проверки эффективности математической модели, описывающей движение больших планет, Луны и Солнца системой дифференциальных уравнений (4), нами проведены исследования движения этих объектов на интервале времени с 1602 по 2193 гг. и результаты вычислений сопоставлены с банком данных DE405. Для исследования развития погрешностей математических моделей методом Эверхарта проведено численное интегрирование уравнений движения данных объектов на более длительный период времени, охватывающий интервал с 7 марта 31 г. до н.э. по 21 октября 3969 г. н.э. [18].

Важно знать, насколько результаты вычислений координат и компонент скоростей планет, Луны и Солнца, полученные с помощью описанных здесь математических моделей, согласуются друг с другом.

Наиболее сложным объектом для исследования движения являются Меркурий и Луна, поэтому из рассматриваемых математических моделей следует отдать предпочтение модели, с помощью которой лучше всего представлено движение этих объектов.

В табл. 1 на интервале времени с 1602 по 2193 гг. представлены координаты и компоненты скоростей Меркурия. Данные координат и компонент скоростей приведены на 0 часов гринвичского времени соответствующей даты, при этом в первой строке - данные банка DE405, во второй строке таблицы - координаты и компоненты скоростей, полученные путем решения уравнений (4).

В табл. 2 приведены элементы орбит Меркурия. Здесь $M-$ средняя аномалия (в градусах), $a$ - большая полуось (в а.е.), $e$ - эксцентриситет, $\omega$ - аргумент перигелия (в градусах), $\Omega$ - долгота восходящего узла (в градусах), $i$ - наклонение (в градусах). В первой строке табл. 2 - элементы орбит Меркурия, найденные по данным банка DE405, во второй и третьей строках полученные путем решения уравнений (4) и (1).

Из сопоставления элементов орбит, найденных с помощью решения уравнений (4) и банка данных DE405, следует, что различие в элементах орбит находится в пределах ошибок наблюдений.

В табл. 3 представлены элементы орбит Меркурия на интервале времени 4000 лет с 7 марта 31 г. до н.э. по 21 октября 3969 г. н.э. В первой, второй и третьей строках - элементы орбит Меркурия, полученные путем решения уравнений (4), (2) и (1) соответственно.

Сравнение координат и элементов орбит Меркурия, полученных с помощью решения уравнений (2) и (4) (см. табл. 1-3), указывает на удовлетво- 


\begin{tabular}{|c|c|c|c|c|c|c|c|c|}
\hline 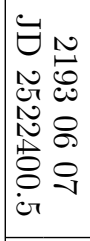 & 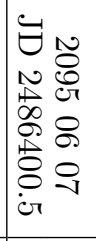 & 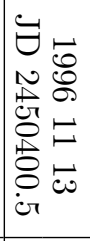 & 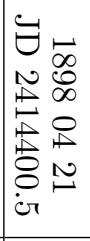 & 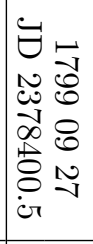 & 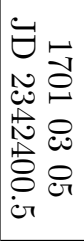 & 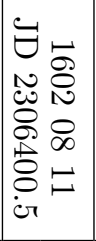 & $\begin{array}{l}2 \\
0 \\
ت \\
0 \\
己 \\
0 \\
\stackrel{0}{0} \\
\stackrel{0}{0}\end{array}$ & 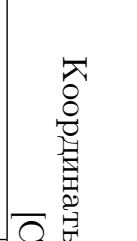 \\
\hline 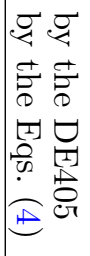 & 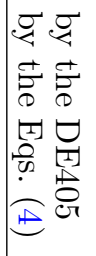 & 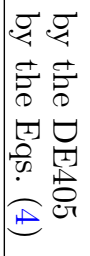 & 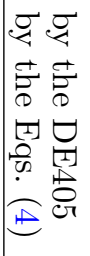 & 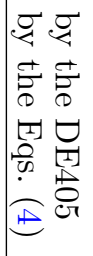 & 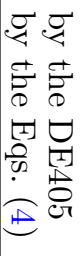 & 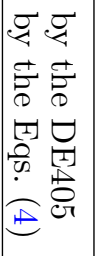 & 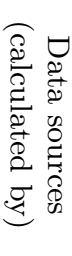 & 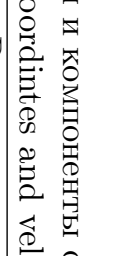 \\
\hline 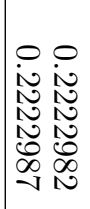 & 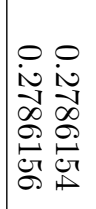 & 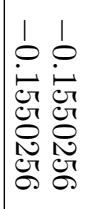 & 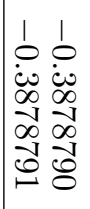 & 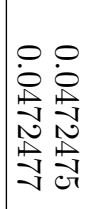 & 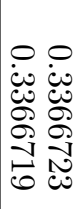 & $\begin{array}{ll}1 & 1 \\
0 & 0 \\
0 & 0 \\
0 & 0 \\
0 & 0 \\
0 & 0 \\
0 & \infty \\
0 & 0 \\
\infty & \infty \\
\infty & 1\end{array}$ & $\begin{array}{l}X \\
\overparen{B} \\
\stackrel{\Xi}{E}\end{array}$ & 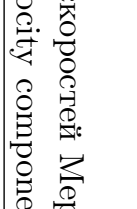 \\
\hline 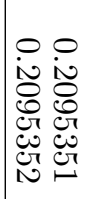 & 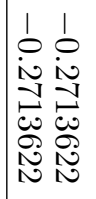 & 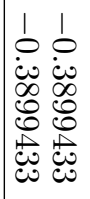 & 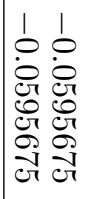 & 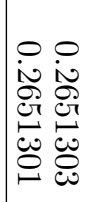 & 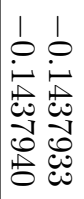 & 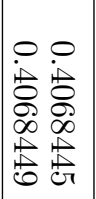 & $\begin{array}{l}\longleftarrow \\
\stackrel{\Xi}{\Xi} \\
\stackrel{\Xi}{E}\end{array}$ & 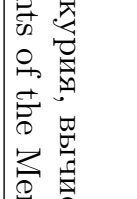 \\
\hline 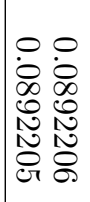 & 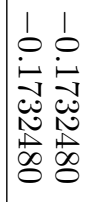 & 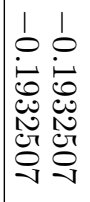 & 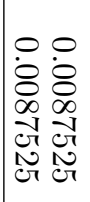 & 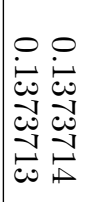 & 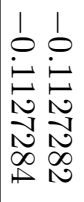 & 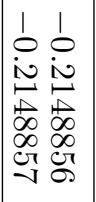 & $\begin{array}{l}\text { N } \\
\stackrel{\Xi}{\Xi} \\
\stackrel{\Xi}{E}\end{array}$ & 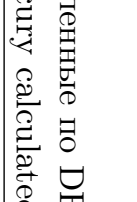 \\
\hline 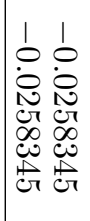 & 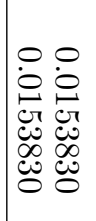 & $\mid \begin{array}{ll}0 & 0 \\
0 & 0 \\
0 & 0 \\
0 & 0 \\
0 & 0 \\
0 & 0 \\
0 & 0 \\
0 & 0 \\
0 & 0 \\
0 & 0\end{array}$ & $\begin{array}{ll}1 & 1 \\
0 & 0 \\
0 & 0 \\
0 & 0 \\
0 & 0 \\
0 & 0 \\
\mathbb{1} & \mathbb{1} \\
0 & 0 \\
\infty & \infty\end{array}$ & 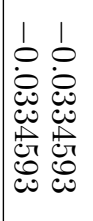 & 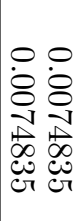 & 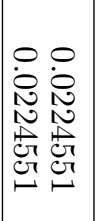 & 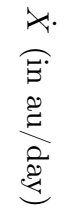 & 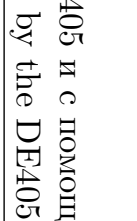 \\
\hline 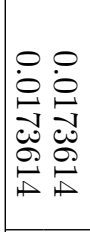 & 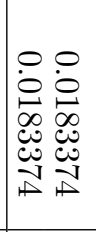 & 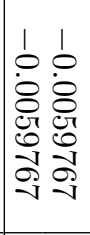 & 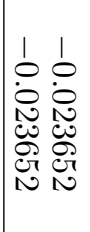 & 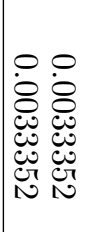 & 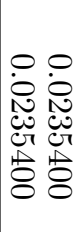 & 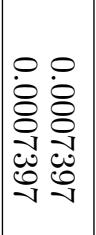 & 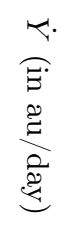 & 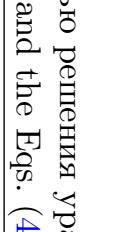 \\
\hline 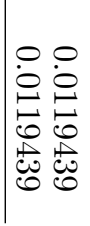 & 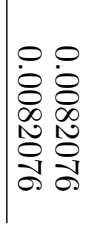 & 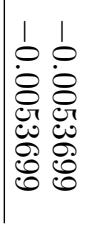 & 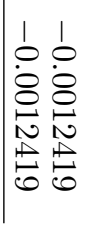 & 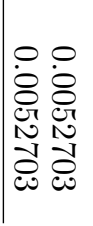 & $\begin{array}{ll}0 & 0 \\
0 & 0 \\
0 & 0 \\
\boxminus & \ddots \\
-1 & 1 \\
0 & 0 \\
0 & 0 \\
ن & 0 \\
0 & 0\end{array}$ & $\mid \begin{array}{ll}1 & 1 \\
0 & 0 \\
0 & 0 \\
0 & 8 \\
0 & 0 \\
0 & 0 \\
0 & 0 \\
8 & 8 \\
8 & 8\end{array}$ & 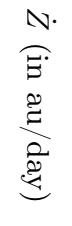 & $\overbrace{\stackrel{\Theta}{\oplus}}^{\stackrel{\Theta}{\Theta}}$ \\
\hline
\end{tabular}




\begin{tabular}{|c|c|c|c|c|c|c|c|c|}
\hline & 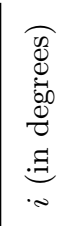 & 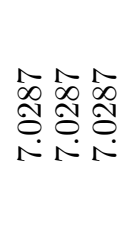 & 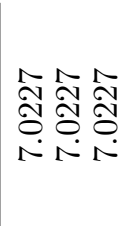 & $\begin{array}{lll}0 & 9 & 0 \\
0 & 0 \\
0 & 0 & 0 \\
1 & 0 & 0 \\
1 & 1\end{array}$ & 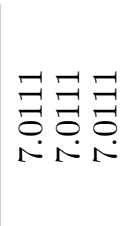 & 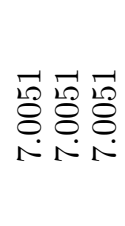 & 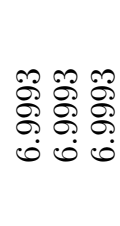 & 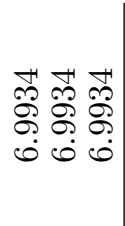 \\
\hline 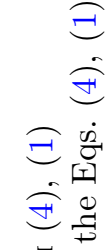 & 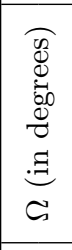 & $\begin{array}{lll}\infty & \infty & \Omega \\
N & N & N \\
N & \sim & \sim \\
\infty & \infty & \infty \\
\infty & \infty & \infty \\
\sim & \infty & +\end{array}$ & 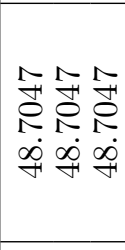 & $\begin{array}{ccc}\infty & \infty & \infty \\
\infty & \infty & \infty \\
\infty & \infty & 1 \\
\infty & 1 & 1 \\
\infty & \infty & \infty \\
\not & \neq & \neq\end{array}$ & 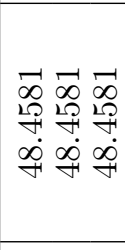 & 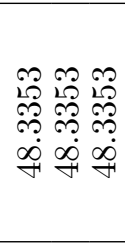 & 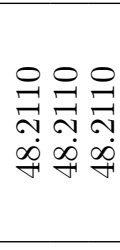 & $\begin{array}{ccc}0 & 0 & 0 \\
0 & 0 & N \\
\infty & \infty & \infty \\
0 & 0 & 0 \\
\infty & \infty & \infty \\
\mathscr{+} & \infty & \infty\end{array}$ \\
\hline 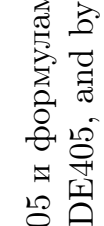 & 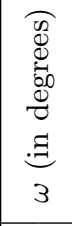 & 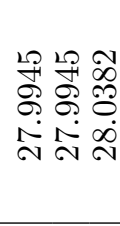 & 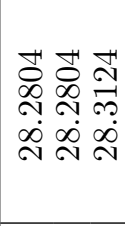 & 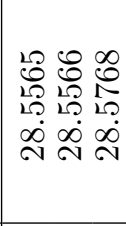 & 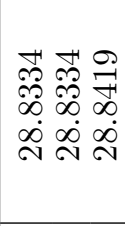 & 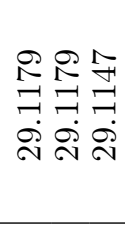 & 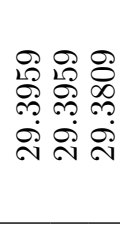 & 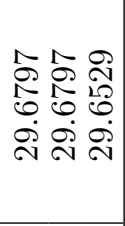 \\
\hline 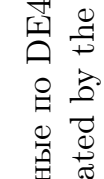 & 0 & 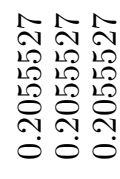 & 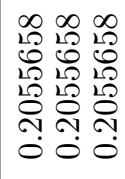 & 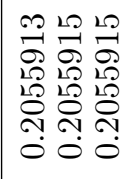 & 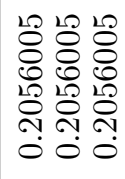 & 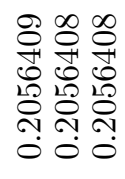 & 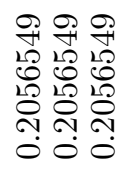 & 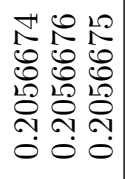 \\
\hline 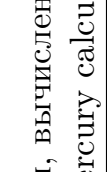 & $\underbrace{\exists}_{0}$ & 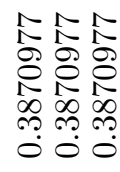 & 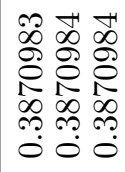 & 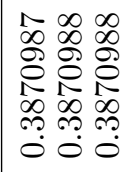 & 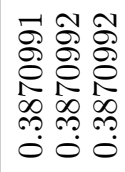 & 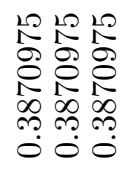 & 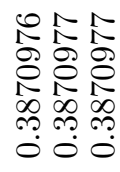 & 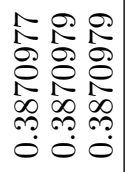 \\
\hline 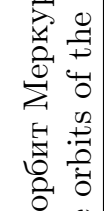 & 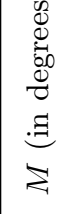 & 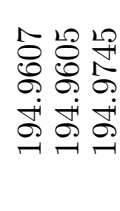 & 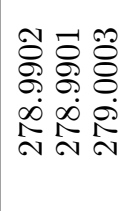 & 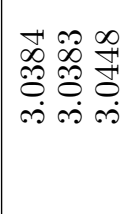 & 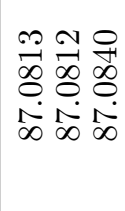 & 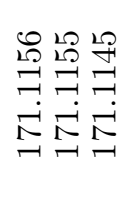 & 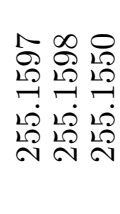 & 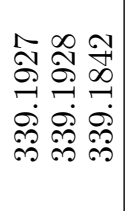 \\
\hline 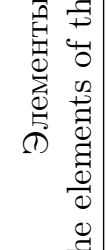 & 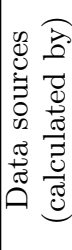 & 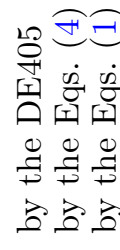 & 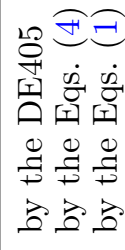 & 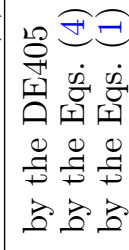 & 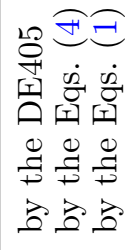 & 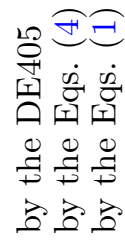 & 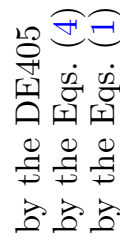 & 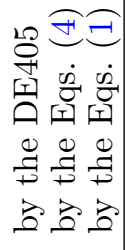 \\
\hline & 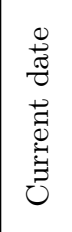 & 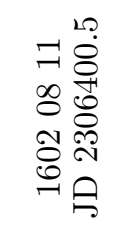 & 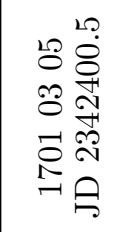 & 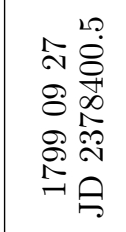 & 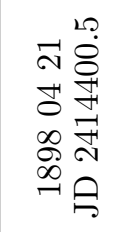 & 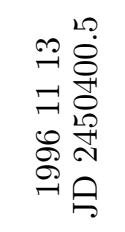 & 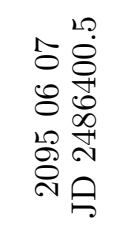 & 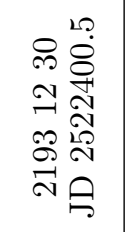 \\
\hline
\end{tabular}




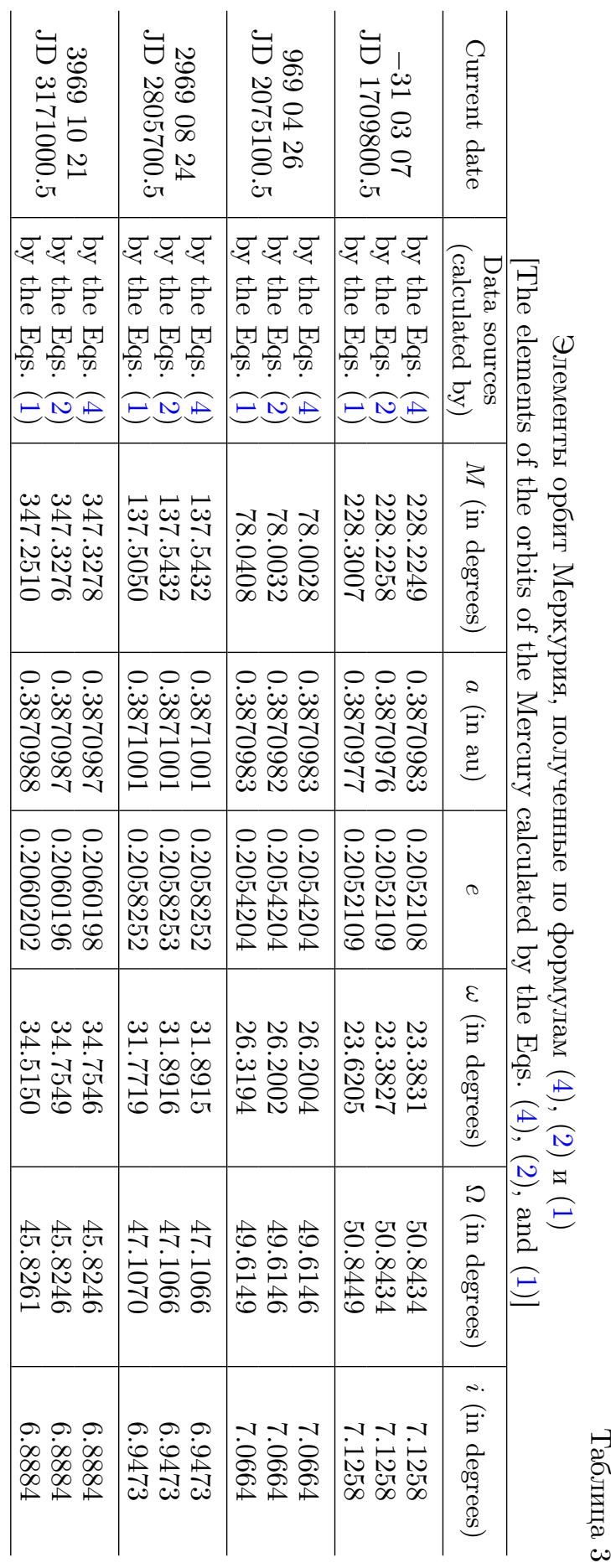


рительное согласие координат и элементов орбит на всем рассматриваемом интервале интегрирования.

Наибольшие расхождения в элементах орбит Меркурия имеет место 7 марта 31 г. до н.э. В средней аномалии оно составляет $0.0009^{\circ}$ (градусов), что соответствует $3^{\prime \prime}$ (секундам дуги); в большой полуоси - 0.0000007 a.e., что соответствует 104.7 км; в аргументе перигелия - $0.0004^{\circ}$, что соответствует $1^{\prime \prime}$; остальные элементы орбит практически совпадают. Невязка векового смещения долготы перигелия Меркурия, найденная путем решения уравнений (4) и (1) составляет 42.92", а при решении уравнений (2) и (1) 42.99". Подобные расхождения в элементах орбит и в вековом смещении перигелия Меркурия, полученных при решении дифференциальных уравнений (4) и (2), не могут быть выявлены с помощью наблюдений, поэтому их можно считать вполне удовлетворительными.

Точность решения релятивистских уравнений существенным образом зависит от выбора системы координат. Для релятивистских уравнений часто используется гармоническая система координат, которая является наиболее подходящей для согласования векового смещения долготы перигелия Меркурия [19-25]. Как показывают проведенные исследования, решение релятивистских уравнений (2) для Меркурия согласуется с решением уравнений (4) на всем исследуемом интервале времени с 31 г. до н.э. по 3969 г. н.э. (см. табл. 1-3). Подобного совпадения решений уравнений (2) и (4) следовало ожидать и для планет Венеры, Земли+Луны и Марса, поскольку их орбиты являются более удаленными от Солнца, чем орбита Меркурия. Однако этого не происходит по причине завышенных скоростей смещения долгот перигелиев этих планет, полученных на основании решения уравнений (2) по сравнению со скоростями, полученными на основании решения уравнений (4).

В табл. 4-9 на интервале времени с 31 г до н.э. по 3969 г н.э. представлены элементы орбит и расхождения в элементах для Венеры, Земли+Луны и Марса, найденные с использованием уравнений (4), уравнений (2), путем совместного решения уравнений (2) и (3) и уравнений (1) на четыре момента времени.

Как следует из проведенных вычислений (см. табл. 4-9), расхождения вековых смещений долгот перигелиев Венеры, Земли+Луны и Марса, полученных с помощью решения уравнений (4) и (1), составляют $2.14^{\prime \prime}, 0.53^{\prime \prime}, 0.07^{\prime \prime}$. Расхождения, найденные с помощью решения уравнений $(2)$ и $(1)$, составляют $8.58^{\prime \prime}, 3.87^{\prime \prime}, 1.33^{\prime \prime}$ соответственно.

В работе [23] отмечается, что ошибки релятивистских поправок в вековом движении перигелиев Венеры и Земли велики и составляют для Венеры $\pm 5.28^{\prime \prime}$, для Земли $\pm 1.79^{\prime \prime}$, для Марса $\pm 0.025^{\prime \prime}$. Расхождения в смещении перигелиев этих планет, найденные с помощью решения уравнений (2) и (4), составляют $6.44^{\prime \prime}, 3.34^{\prime \prime}, 1.26^{\prime \prime}$ соответственно. Как следует из результатов вычислений, вековые смещения долгот перигелиев Венеры, Земли+Луны и Марса, найденных путем решения уравнений (4) и (2), различаются на $6.44^{\prime \prime}$, $3.34^{\prime \prime}, 1.26^{\prime \prime}$ соответственно. Хотя эти различия незначительные и на ограниченных интервалах времени в пределах столетия их трудно обнаружить с помощью наблюдений, с течением времени в силу векового характера движения перигелиев Венеры, Земли +Луны и Марса, имеют место заметные различия элементов орбит, найденных с помощью решений уравнений (4) и (2). 


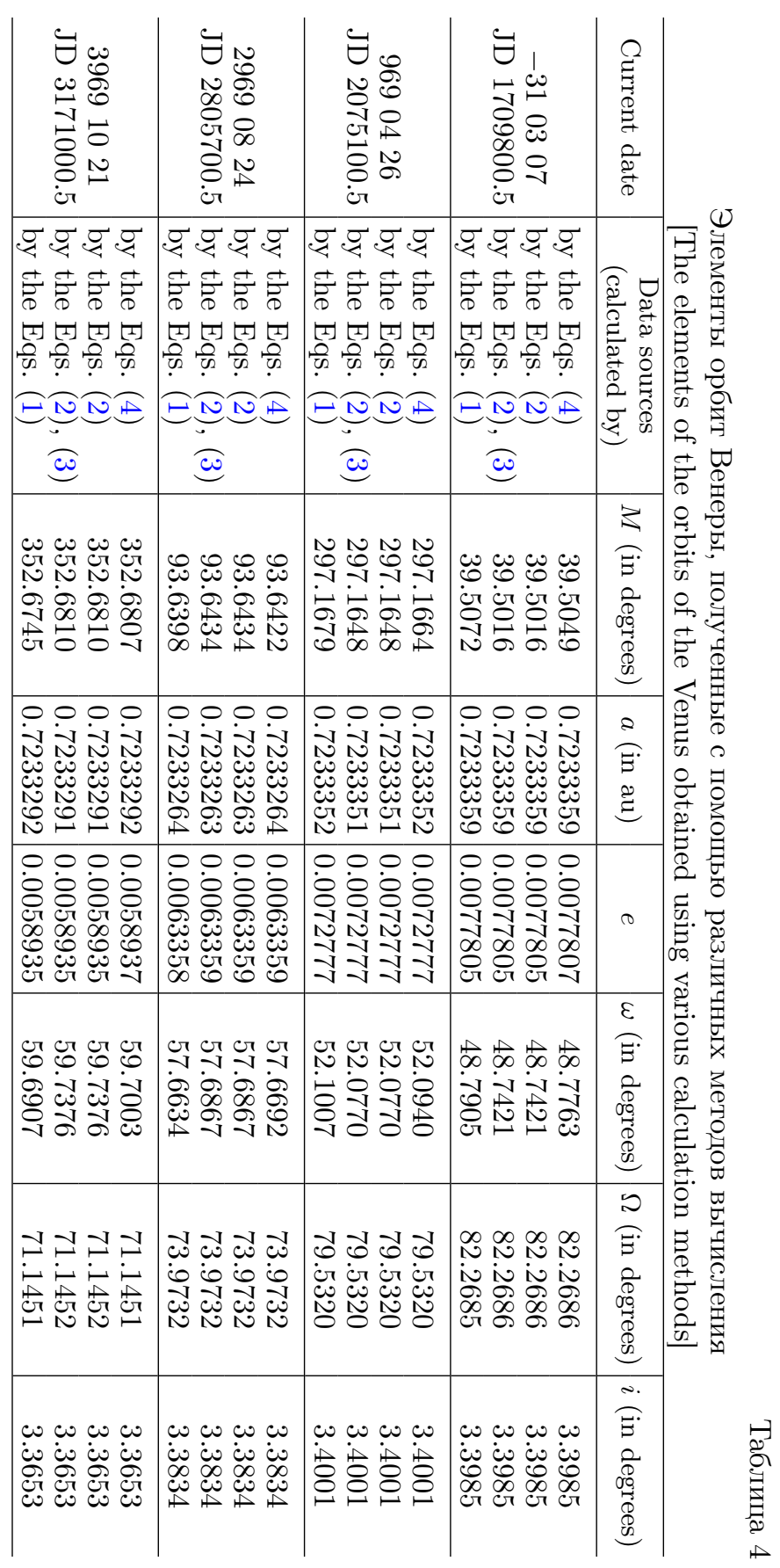




\begin{tabular}{|c|c|c|c|c|c|}
\hline 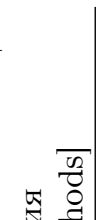 & $\begin{array}{l}0 \\
0 \\
0 \\
0 \\
0 \\
0 \\
.0 \\
. \\
. \infty\end{array}$ & 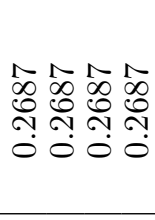 & 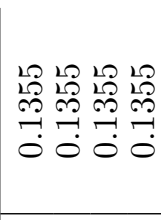 & 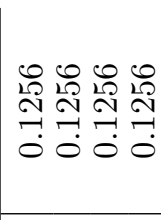 & 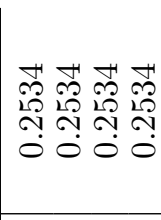 \\
\hline 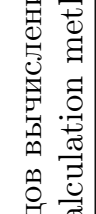 & 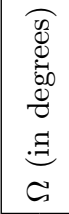 & 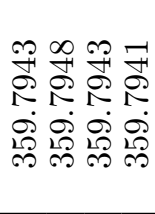 & 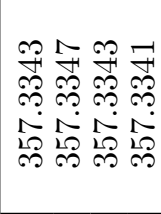 & 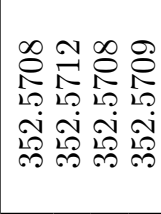 & 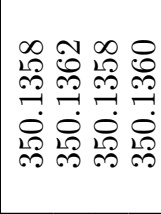 \\
\hline 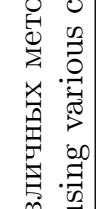 & 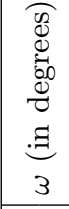 & 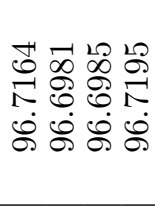 & 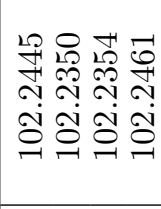 & 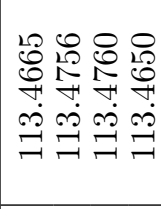 & 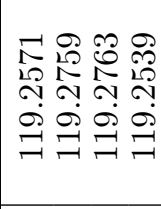 \\
\hline 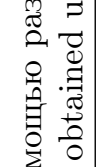 & 0 & 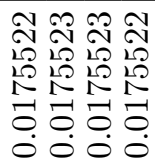 & 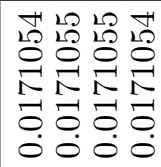 & 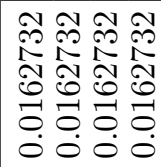 & 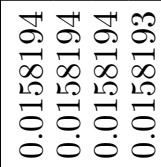 \\
\hline 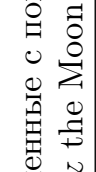 & $\begin{array}{l}\underset{\sigma}{\sigma} \\
\Xi \\
0\end{array}$ & 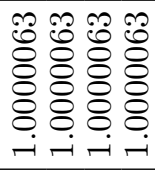 & 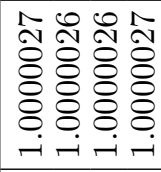 & 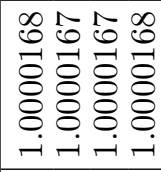 & 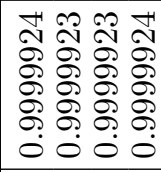 \\
\hline 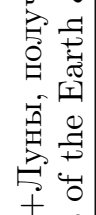 & 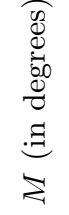 & 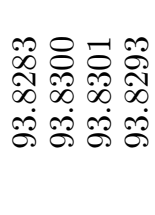 & 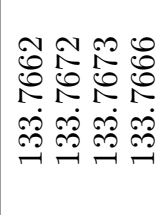 & 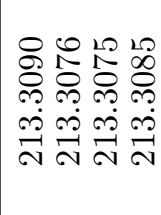 & 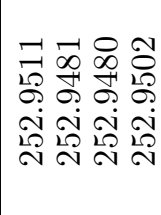 \\
\hline 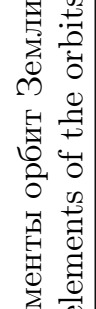 & 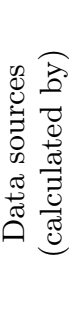 & 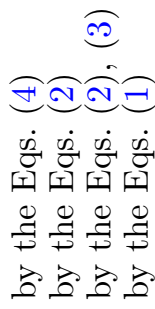 & 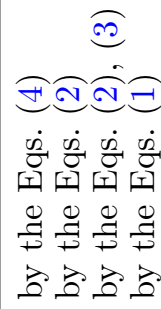 & 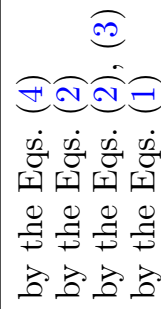 & 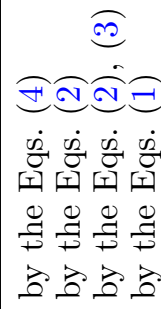 \\
\hline$\stackrel{\vec{D}}{\stackrel{g}{E}}$ & 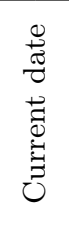 & 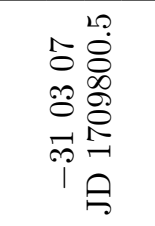 & 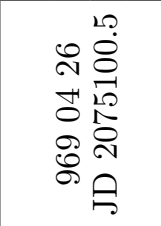 & 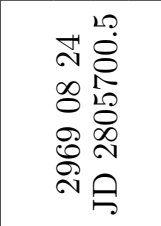 & 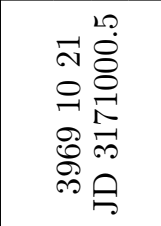 \\
\hline
\end{tabular}




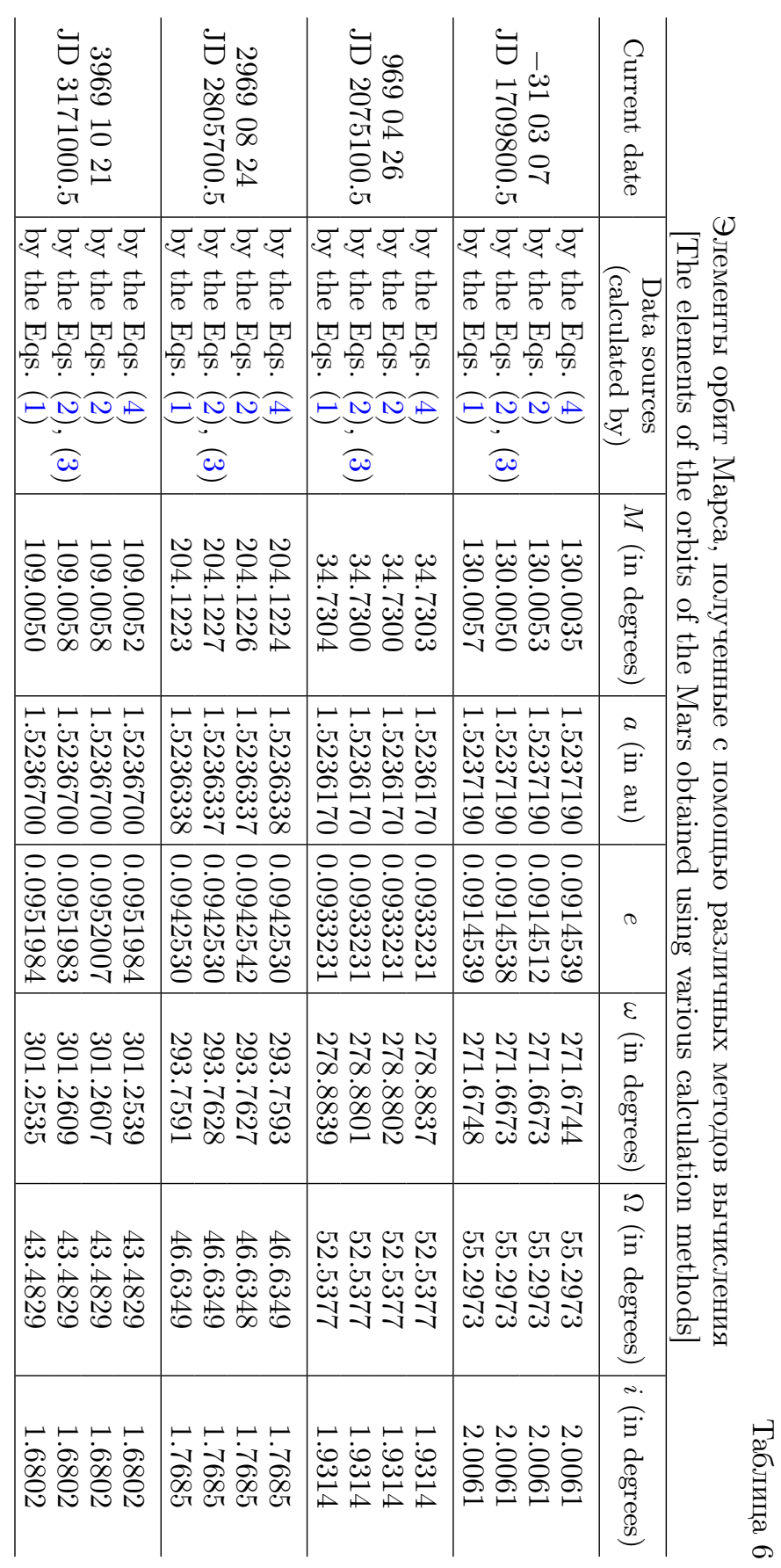




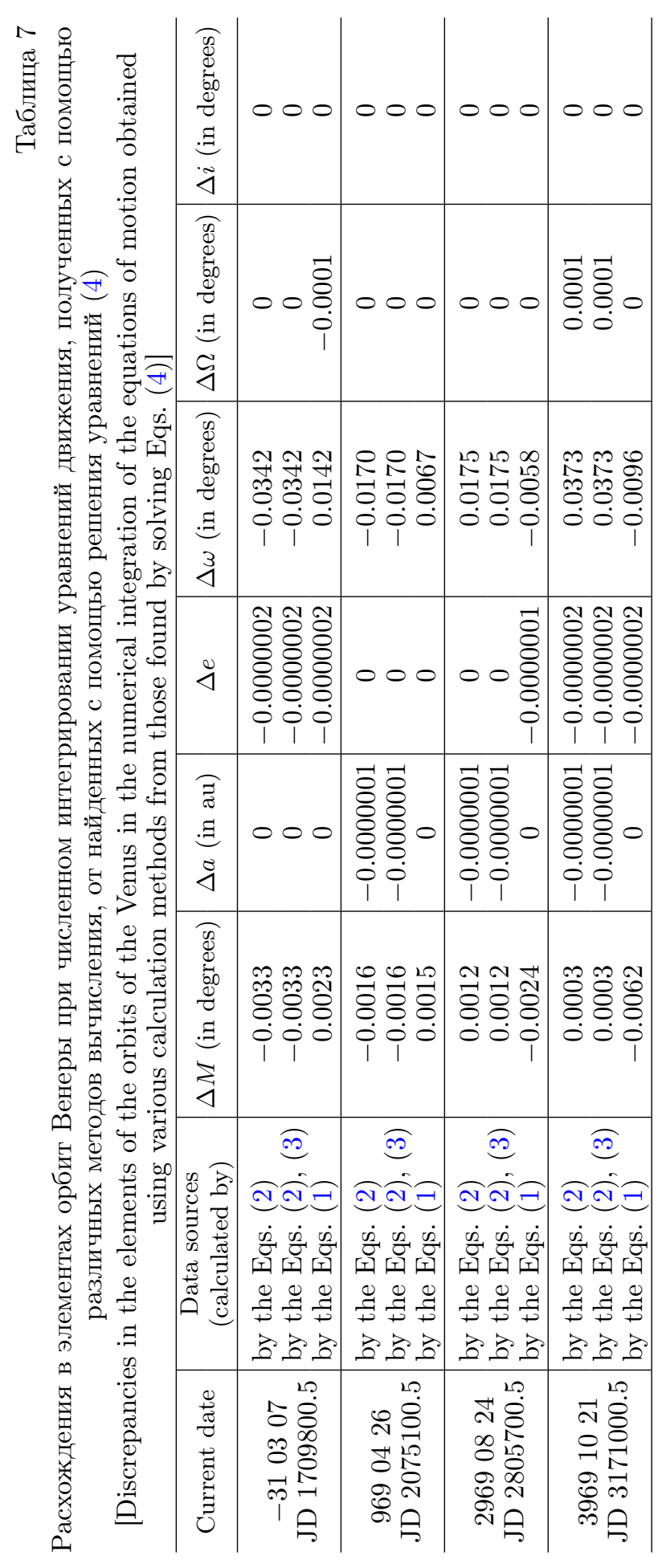




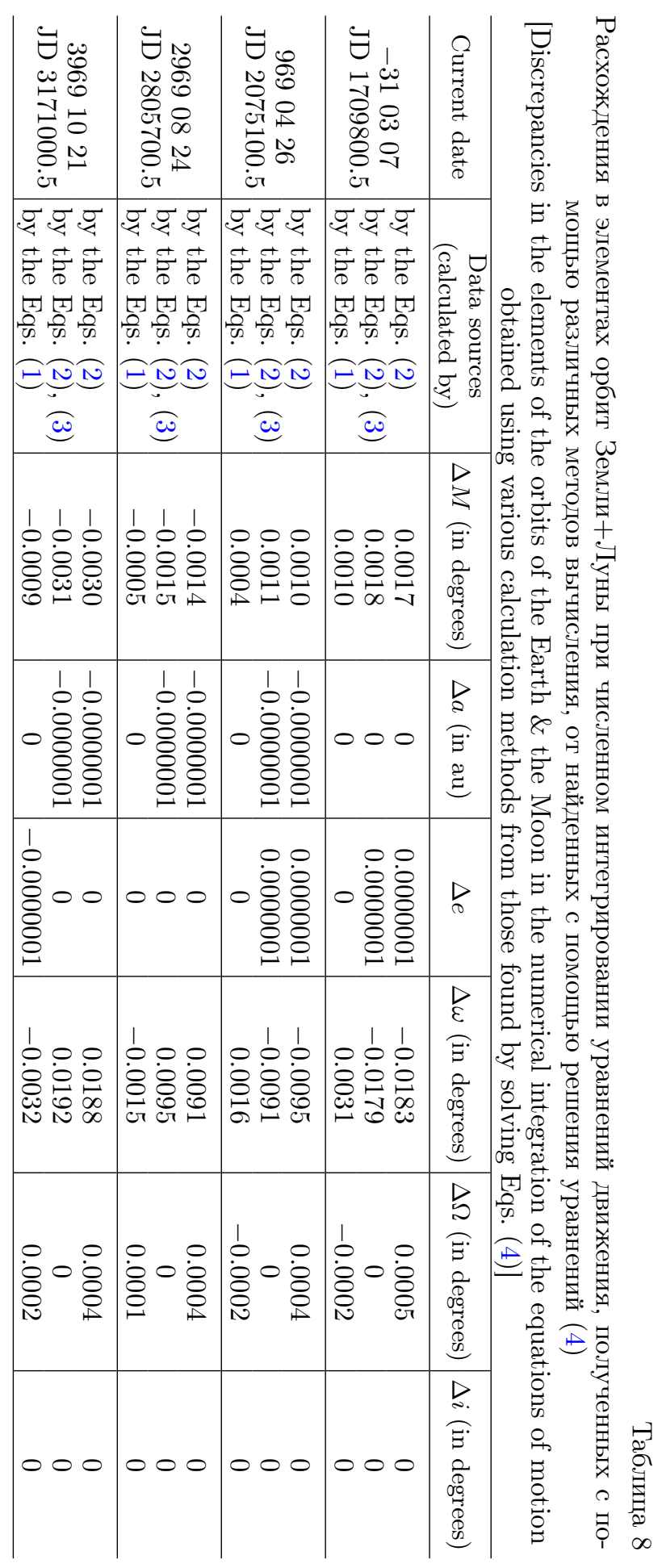




\begin{tabular}{|c|c|c|c|c|c|}
\hline 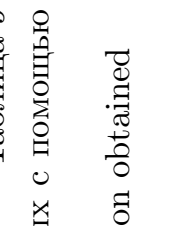 & 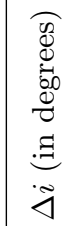 & 000 & 000 & 000 & 000 \\
\hline 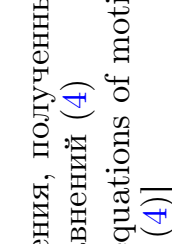 & 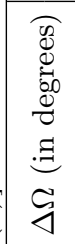 & 000 & 000 & 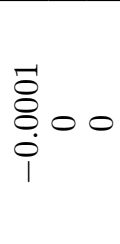 & 000 \\
\hline 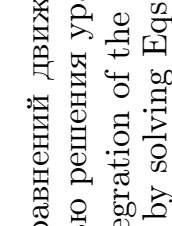 & 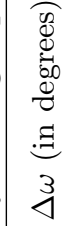 & 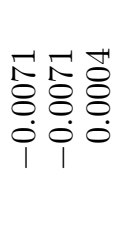 & 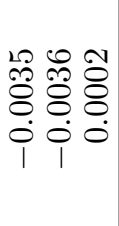 & 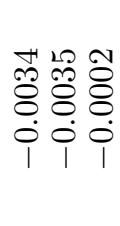 & 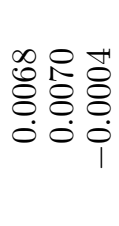 \\
\hline 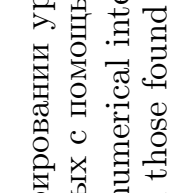 & 4 & 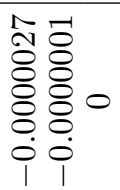 & 000 & 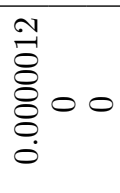 & 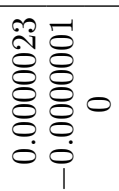 \\
\hline 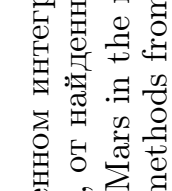 & 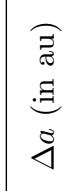 & 000 & 000 & 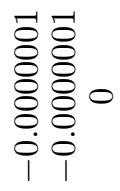 & 000 \\
\hline 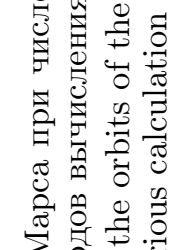 & 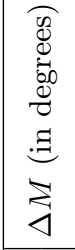 & 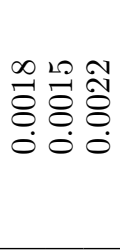 & 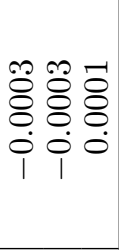 & 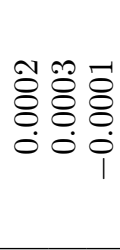 & $\begin{array}{l}\mathscr{Q} \\
\varrho\end{array}$ \\
\hline 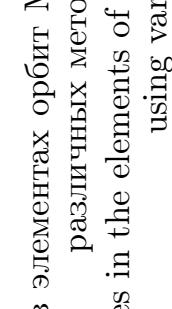 & 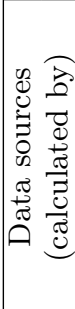 & 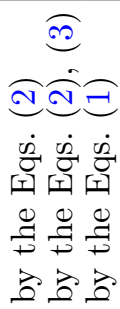 & 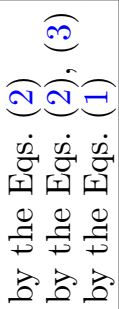 & 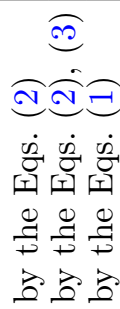 & 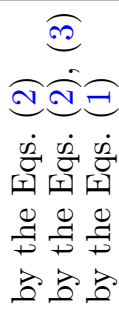 \\
\hline 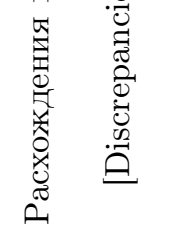 & 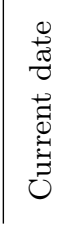 & 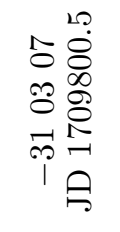 & 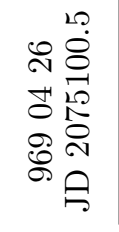 & 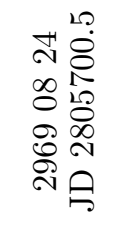 & 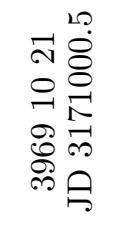 \\
\hline
\end{tabular}


Наибольшие отклонения наблюдаются в средних аномалиях и аргументах перигелиев. Как показывают результаты вычислений (см. табл. 4-6 и 79), расхождения в средней аномалии у Венеры, Земли+Луны и Марса составляют, соответственно, $\Delta M=12^{\prime \prime}, 6^{\prime \prime}$ и $6^{\prime \prime}$, а в аргументах перигелиев$\Delta \omega=-0.0342^{\circ},-0.0183^{\circ}$ и $-0.0071^{\circ}$ в 31 г. до н.э. В 3969 г. расхождения в средней аномалии у Венеры, Земли+Луны и Марса достигнут $\Delta \omega=0.0373^{\circ}$, $0.0188^{\circ}$ и $0.0068^{\circ}$ соответственно. С увеличением интервала интегрирования эти отклонения будут увеличиваться, что непосредственно отразится на расхождении элементов орбит планет, найденных с помощью решения уравнений (4) и (2).

Проведенные исследования показывают, что решение релятивистских уравнений не обеспечивает одинаковой точности для всех объектов. По этой причине прогнозирование движений Венеры, Земли +Луны и Марса путем решения уравнений (2) не является таким же точными, как для Меркурия. Для обоснования данного предположения необходимо показать преимущество использования уравнений (4) по сравнению с уравнениями (2) при исследовании движения этих небесных тел. Для сравнения эффективности использования уравнений (4) и (2) наиболее подходящим объектом для исследования является Луна.

Геоцентрические координаты и компоненты скоростей Луны, найденные путем решения уравнений (4) и с помощью банка данных DE405, приведены в табл. 10.

Следует отметить, что для согласования геоцентрических координат Луны с наблюдениями при создании DE405 ее авторы наряду с уравнениями (2) решают совместно уравнения, учитывающие форму Луны и Земли, а также земные приливы. Подобный прием, учитывающий форму небесных тел и приливные взаимодействия, не является строго обоснованным, т.к. эти уравнения получены на основе ньютоновой теории. Кроме того, данные уравнения содержат много свободных параметров, значения которых определены с невысокой точностью. При согласовании численной теории движения больших планет и Луны DE405 с наблюдениями происходит существенное усложнение релятивистской модели движения небесных тел по сравнению с ньютоновой моделью.

Из результатов вычислений, представленных в табл. 10, следует, что координаты и скорости Луны, полученные путем решения уравнений (4), в основе которых лежит принцип взаимодействия окружающего пространства с движущимся материальным телом, отличаются от данных DE405 несущественно на интервале времени \pm 100 лет от начального момента интегрирования. Например (см. табл. 10), 21 апреля 1898 г. и 13 ноября 1996 г. максимальное отклонение в координатах, найденных с помощью DE405 и полученных с помощью решения уравнений (4), составляет 0.0000003 а.е., что соответствует 45 км. Отклонение компонент скоростей также незначительное, не превышающее 0.0000001 а.е./сут. или $1.7 \cdot 10^{-4} \mathrm{kM} /$ сек. Максимальное расхождение двух методов для геоцентрических координат Луны имеет место 11 августа 1602 г. При этом различия в вычислениях составляют следующие величины: $\Delta X=0.0000003$ a.e., $\Delta Y=0.0000006$ a.e. $\Delta Z=-0.0000021$ a.e., что соответствует $\Delta X=45 \mathrm{kм}, \Delta Y=90$ км и $\Delta Z=-314$ км. Максимальное расхождение компонент скоростей - 0.0000002 а.е./сут. Полученные отклонения 


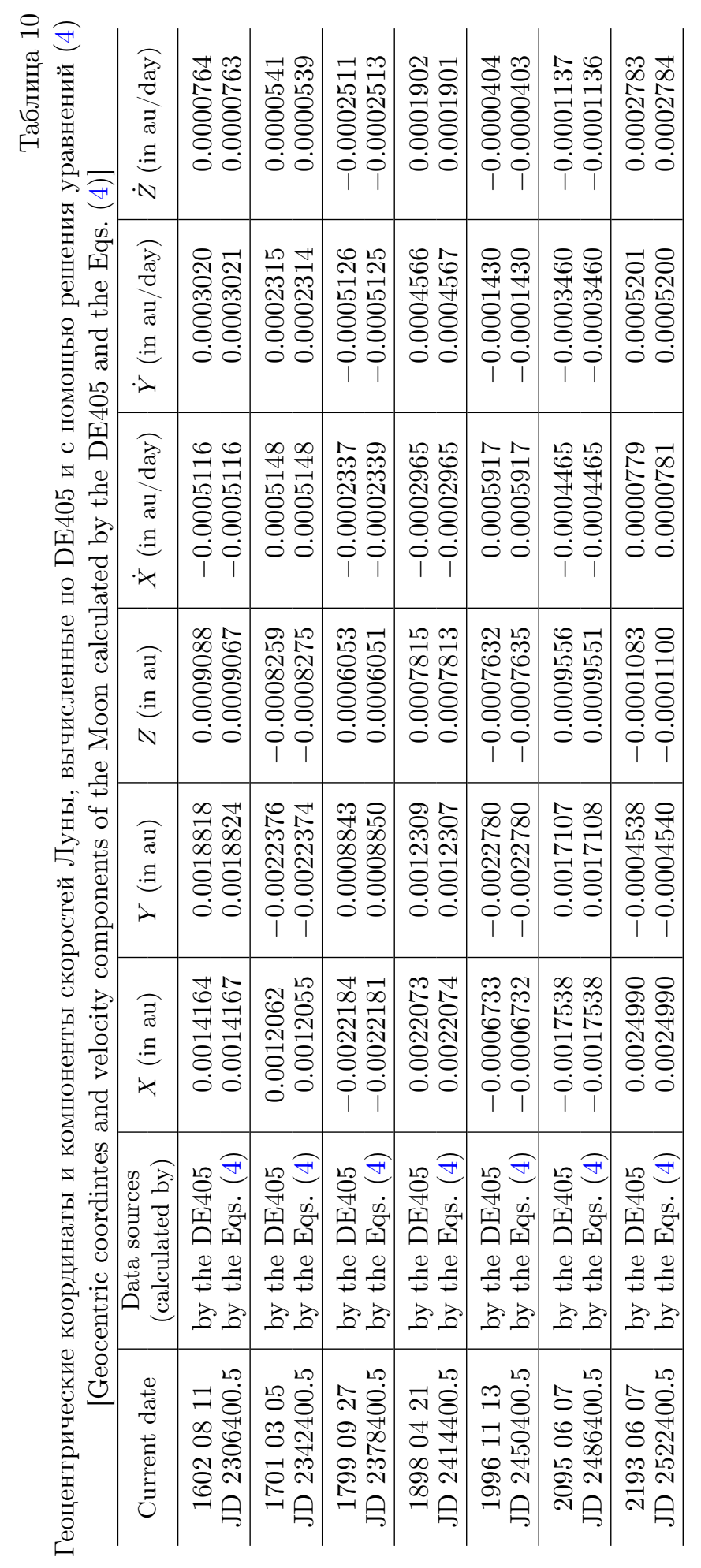


в геоцентрических координатах и компонентах скоростей Луны находятся в пределах ошибок как оптических, так и радиолокационных наблюдений, поэтому можно сказать, что при определении геоцентрических координат и компонент скоростей Луны с помощью банка данных DE405 и путем решения уравнений (4) результаты на всем интервале интегрирования получаются практически с одинаковой точностью.

В табл. 11 приведены геоцентрические координаты и скорости Луны, вычисленные с учетом релятивистских эффектов и ньютоновых уравнений, но без учета фигуры Земли, т.е. получены путем решения дифференциальных уравнений движения (2) и уравнений (1). Сопоставление данных, приведенных в табл. 10 и 11, показывает, что на интервале времени \pm 100 лет от начального момента интегрирования расхождения координат, найденных путем решения уравнений (2) и с помощью банка данных DE405, значительно превышают расхождения координат, полученных с помощью решения уравнений (4) и банка данных DE405. Так, на момент 21 апреля 1898 г. максимальное отклонение в координатах, найденных с помощью DE405 и полученных с помощью решения уравнений (2), составляет 0.0000035 а.е., что соответствует 554 км. Отклонение компонент скоростей также значительное и равно 0.0000008 a.e./сут., или $6.9 \cdot 10^{-4} \mathrm{kM} /$ сек.

Из сравнения данных, приведенных в табл. 12 и 13, следует, что наибольшие расхождения координат, найденных путем решения уравнений (2) и с помощью банка данных DE405, имеют место 11 августа 1602 г. и составляют $\Delta X=-0.000019$ a.e., $\Delta Y=0.0000128$ a.е. и $\Delta Z=0.0000012$ a.е., что соответствует $\Delta X=-2887 \mathrm{kм}, \Delta Y=1915$ км и $\Delta Z=180$ км. Аналогичные расхождения координат, найденных с помощью решения уравнений (1) и DE405 (см. табл. 12 и 13), составляют $\Delta X=-0.0000267$ a.e., $\Delta Y=-0.0000174$ a.e. и $\Delta Z=-0.0000023$ а.е., что соответствует $\Delta X=-3994 \mathrm{kм}, \Delta Y=2603 \mathrm{kм}$ и $\Delta Z=344 \mathrm{kм}$.

Из проведенного сопоставления следует, что расхождения координат Луны, полученные на основе банка данных DE405 и путем решения дифференциальных уравнений движения (1) и (2), превышают более чем на порядок аналогичные расхождения координат Луны, полученные с помощью DE405 и на основании решения уравнений (4). Проведенные исследования указывают на ограниченную возможность ньютоновых и релятивистских уравнений без совместного решения дополнительных уравнений (3) для исследования движения Луны на интервале времени порядка нескольких столетий.

В табл. 14 приведены геоцентрические координаты Луны, полученные с помощью решения дифференциальных уравнений (4) и путем совместного решения уравнений (2) и (3), а также уравнений (2) и (1). В первой строке табл. 14 находятся координаты Луны, найденные с помощью совместного решения уравнений (2) и (3), во второй, третьей и четвертой - координаты, полученные с помощью решения уравнений (4), (2) и (1).

В табл. 15 и 16 представлены расхождения геоцентрических координат и компонент скоростей Луны, полученных с помощью математических моделей (4), (2) и (1), от координат, найденных путем совместного решения уравнений (2) и (3). В первой строке табл. 15 и 16 находятся расхождения координат Луны, найденных с помощью совместного решения уравнений (2) и (3), во второй строке - координат, полученных с помощью решения диффе- 


\begin{tabular}{|c|c|c|c|c|c|c|c|c|}
\hline$\widetilde{N}$ & 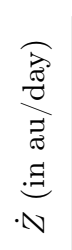 & \begin{tabular}{l} 
ㄱ \\
\multirow{1}{*}{} \\
8 \\
8 \\
8 \\
8 \\
8 \\
0 \\
0
\end{tabular} & $\begin{array}{l}1 \\
10 \\
10 \\
8 \\
8 \\
8 \\
8 \\
8 \\
0 \\
0\end{array}$ & 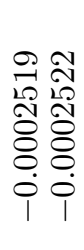 & \begin{tabular}{l}
$\infty$ \\
$\infty$ \\
$\infty$ \\
$\infty$ \\
\hdashline \\
8 \\
8 \\
8 \\
8 \\
0 \\
0 \\
0
\end{tabular} & $\begin{array}{ll}2 & 12 \\
O & 2 \\
& 9 \\
8 & 8 \\
0 & 8 \\
8 & 8 \\
0 & 0 \\
0 & 0 \\
1 & 1\end{array}$ & 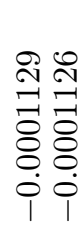 & 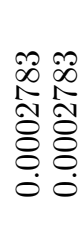 \\
\hline 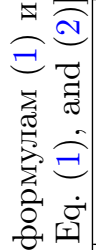 & 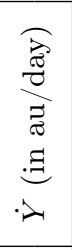 & 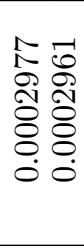 & 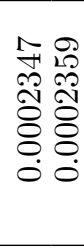 & 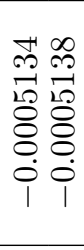 & $\begin{array}{l}1 \\
0 \\
0 \\
10 \\
10 \\
8 \\
8 \\
8 \\
8 \\
0 \\
0 \\
0\end{array}$ & 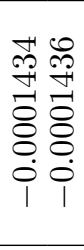 & 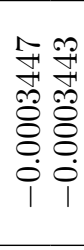 & 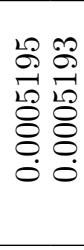 \\
\hline 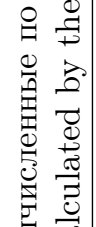 & 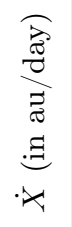 & $\begin{array}{ll}0 & \infty \\
2 & 10 \\
20 & 10 \\
8 & 8 \\
8 & 8 \\
0 & 0 \\
0 & 0 \\
1 & 1\end{array}$ & 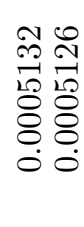 & 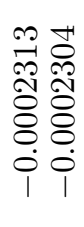 & 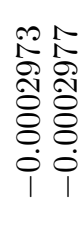 & 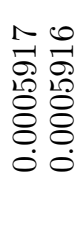 & 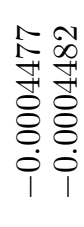 & 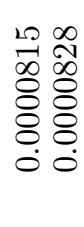 \\
\hline 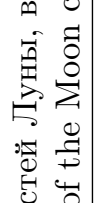 & 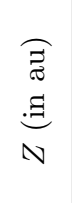 & $\begin{array}{l}8= \\
8= \\
\sigma \\
\delta \\
8 \\
8 \\
8 \\
0 \\
0 \\
0\end{array}$ & 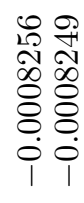 & 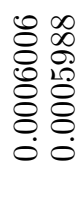 & 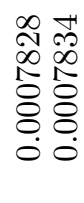 & 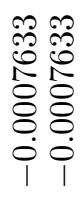 & $\begin{array}{l}R \\
1 \\
12 \\
2 \\
8 \\
8 \\
8 \\
8 \\
8 \\
0 \\
0\end{array}$ & 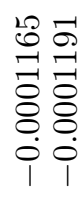 \\
\hline 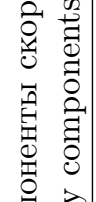 & 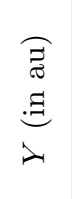 & 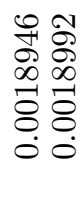 & 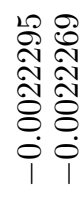 & \begin{tabular}{ll}
0 & 0 \\
1 & \multicolumn{1}{|c}{} \\
$\infty$ & 0 \\
0 & 8 \\
8 & 8 \\
0 & 8 \\
0 & 0
\end{tabular} & 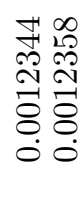 & 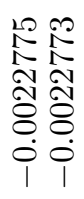 & 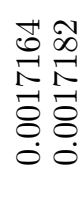 & $\begin{array}{ll}0 & 0 \\
0 & 8 \\
0 & ? \\
0 & 0 \\
8 & 8 \\
8 & 8 \\
0 & 0 \\
0 & 0 \\
1 & 1\end{array}$ \\
\hline 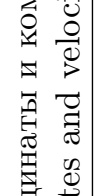 & 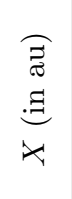 & 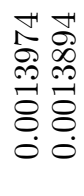 & 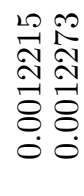 & 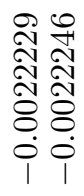 & 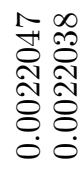 & \begin{tabular}{ll}
0 & 0 \\
\multirow{7}{1}{} & 10 \\
0 & 1 \\
0 & 0 \\
8 & 8 \\
0 & 8 \\
0 & 0 \\
0 & 0 \\
1 & 1
\end{tabular} & 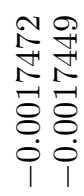 & 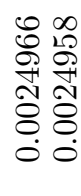 \\
\hline 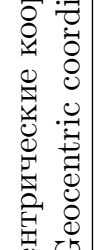 & 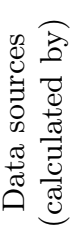 & 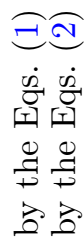 & 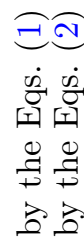 & 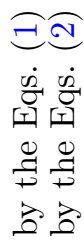 & 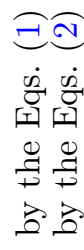 & 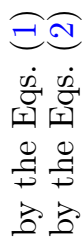 & 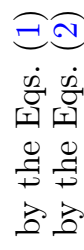 & 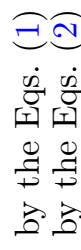 \\
\hline Q & 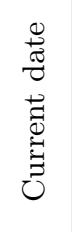 & 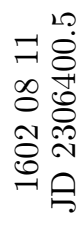 & 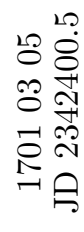 & 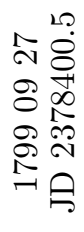 & 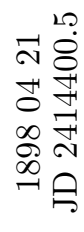 & 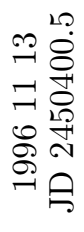 & 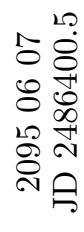 & 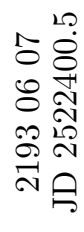 \\
\hline
\end{tabular}




\begin{tabular}{|c|c|c|c|c|c|c|c|c|}
\hline 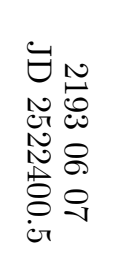 & 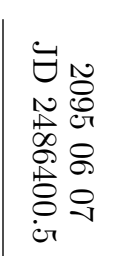 & 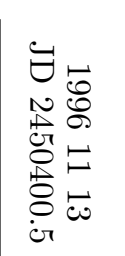 & 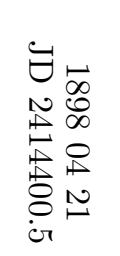 & 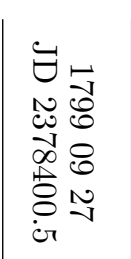 & 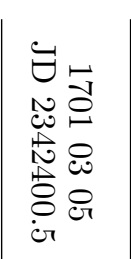 & 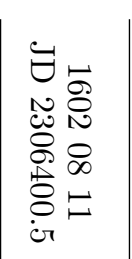 & 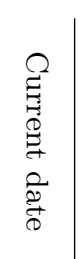 & 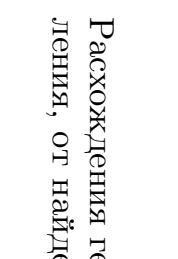 \\
\hline 屈 & एृ & 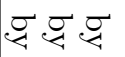 & 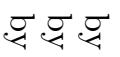 & ত্ব & $\sigma_{\psi} \sigma \sigma_{4}$ & 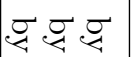 & & \\
\hline 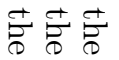 & 宥㝒㝒 & 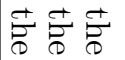 & 宕㝒完 & 穴奋奋 & 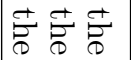 & 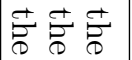 & 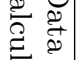 & \\
\hline $\begin{array}{l}1 \\
\text { की }\end{array}$ & 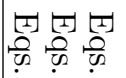 & 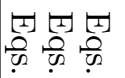 & 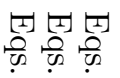 & 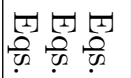 & 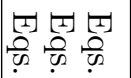 & $\mid \begin{array}{ll}x \\
\text { की } \\
\text { की }\end{array}$ & 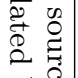 & 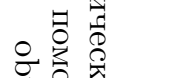 \\
\hline 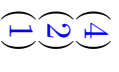 & $\overparen{\Theta} \mathbb{E}$ & 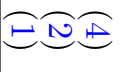 & $\overparen{E} \mathbb{E}$ & $\overparen{E} \mathbb{E}$ & $\bigoplus \overparen{E}$ & $\overparen{E} \mathbb{E}$ & & \\
\hline 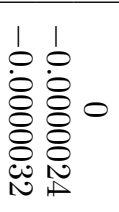 & 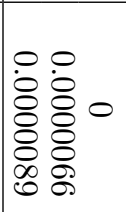 & $\begin{array}{lll}1 & 1 & \\
0 & 0 & 0 \\
0 & 0 & 8 \\
8 & 8 & 8 \\
8 & 8 & 8 \\
8 & 8 & 8 \\
8 & 8 & 8 \\
0 & 0 & 8 \\
1 & 0 & 0\end{array}$ & 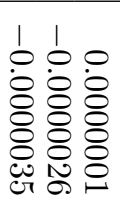 & 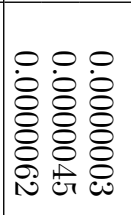 & 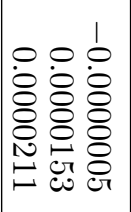 & \begin{tabular}{lll}
1 & 1 & 0 \\
0 & 0 & 0 \\
0 & 0 & 0 \\
8 & 0 & 8 \\
0 & 8 & 8 \\
0 & 8 & 8 \\
0 & 0 & 8 \\
0 & 0 & 0 \\
\hdashline & 0 & 0
\end{tabular} & 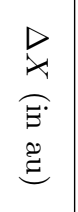 & 焉 \\
\hline 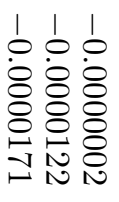 & 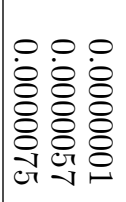 & 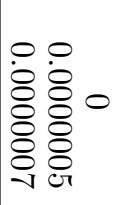 & 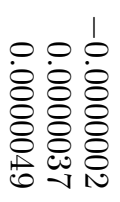 & 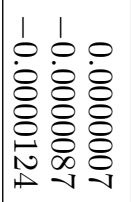 & 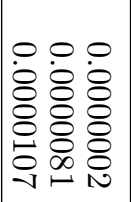 & 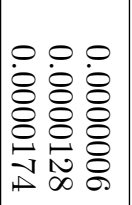 & $\begin{array}{l}\text { ¿ } \\
\text { E. } \\
\text { है }\end{array}$ & $=\infty$ \\
\hline $\begin{array}{lll}1 & 1 & 1 \\
0 & 0 & 0 \\
8 & 8 & 0 \\
8 & 8 & 8 \\
8 & 8 & 8 \\
0 & 8 & 8 \\
0 & 0 & 0 \\
0 & \infty & 0 \\
\infty & 0 & \ddots\end{array}$ & 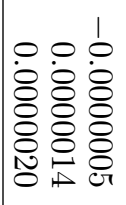 & $\begin{array}{lll}1 & 1 & \\
0 & 0 & 0 \\
8 & 0 & 0 \\
8 & 8 & 8 \\
8 & 8 & 8 \\
8 & 8 & 8 \\
8 & 8 & 8 \\
0 & 0 & 0\end{array}$ & 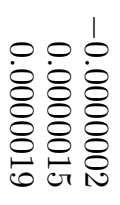 & 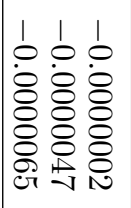 & $\begin{array}{lll}0 & 0 & 1 \\
0 & 0 & 0 \\
8 & 8 & 8 \\
8 & 8 & 8 \\
8 & 8 & 8 \\
8 & 8 & 8 \\
\wp & 8 & \varrho \\
0 & 0 & \ddots\end{array}$ & 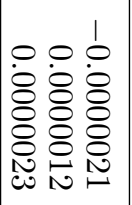 & $\begin{array}{l}\text { N } \\
\text { E. } \\
\text { ¿ }\end{array}$ & $\begin{array}{l}\text { क } \\
\Leftrightarrow\end{array}$ \\
\hline 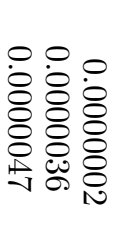 & 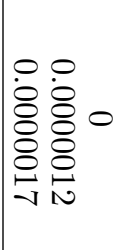 & 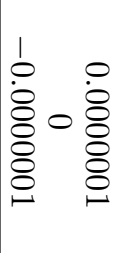 & 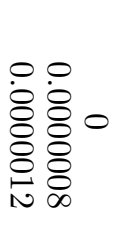 & 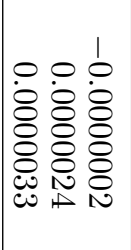 & $\begin{array}{ll}1 & 1 \\
0 & 0 \\
0 & 0 \\
8 & 8 \\
8 & 8 \\
8 & 8 \\
0 & 0 \\
0 & 0 \\
N & 0\end{array}$ & 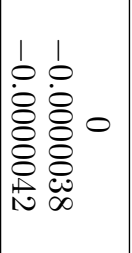 & 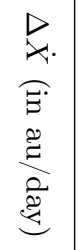 & 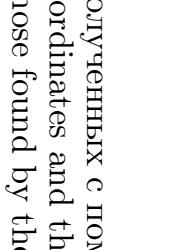 \\
\hline 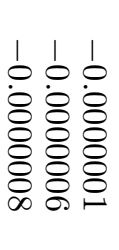 & 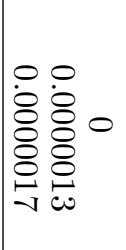 & $\begin{array}{ll}1 & 1 \\
0 & 0 \\
0 & 8 \\
8 & 8 \\
8 & 8 \\
8 & 8 \\
8 & 8 \\
8 & 8 \\
8 & 0 \\
0 & 1\end{array}$ & $\begin{array}{lll}1 & 1 & 0 \\
0 & 0 & 0 \\
8 & 8 & 8 \\
8 & 8 & 8 \\
8 & 8 & 8 \\
8 & 8 & 8 \\
8 & 8 & 8 \\
8 & 8 & 0\end{array}$ & 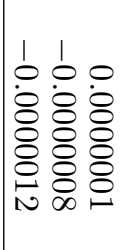 & 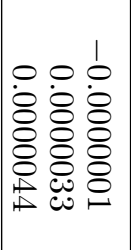 & 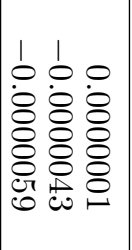 & 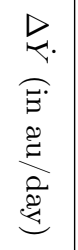 & $\begin{array}{rl} & \\
0 & 0 \\
0 & 0 \\
0 & 0 \\
0 \\
0 \\
0 \\
0 \\
0\end{array}$ \\
\hline 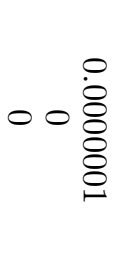 & 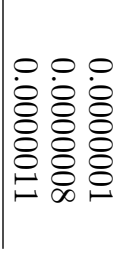 & 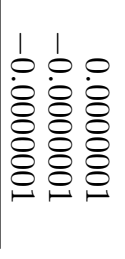 & \begin{tabular}{lll}
1 & 1 & 1 \\
0 & 0 & 0 \\
0 & 0 & 0 \\
8 & 8 & 8 \\
8 & 8 & 8 \\
8 & 8 & 8 \\
8 & 8 & 8 \\
8 & 8 & 8 \\
\hdashline & 0 & 0
\end{tabular} & $\begin{array}{lll}1 & 1 & 1 \\
0 & 0 & 0 \\
0 & 0 & 8 \\
8 & 8 & 8 \\
8 & 8 & 8 \\
8 & 8 & 8 \\
8 & 8 & 8 \\
ٍ & 8 & 8 \\
& \infty & 0\end{array}$ & 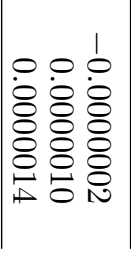 & $\begin{array}{lll}1 & 1 & 1 \\
0 & 0 & 0 \\
0 & 0 & 0 \\
8 & 8 & 8 \\
8 & 8 & 8 \\
8 & 8 & 8 \\
8 & 8 & 8 \\
0 & 0 & 0 \\
0 & 0 & 0\end{array}$ & 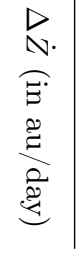 & ถั่ \\
\hline
\end{tabular}




\begin{tabular}{|c|c|c|c|c|c|c|c|c|}
\hline \multicolumn{9}{|c|}{ 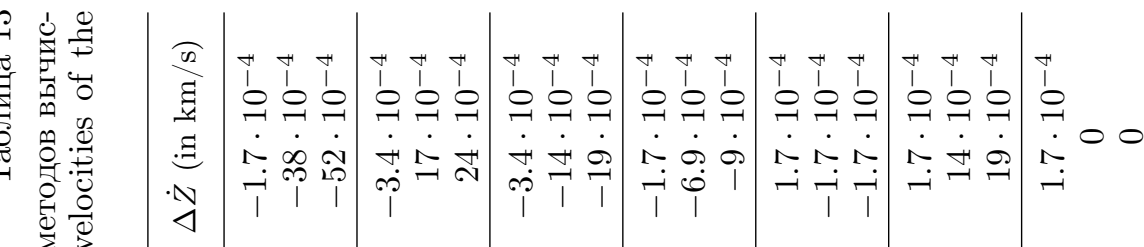 } \\
\hline 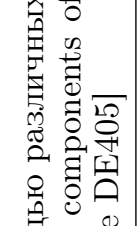 & 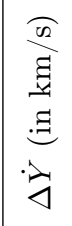 & 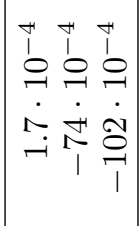 & 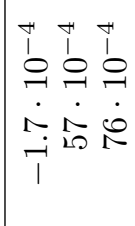 & 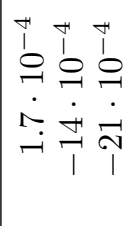 & 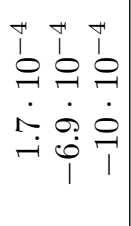 & 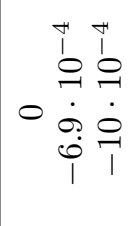 & 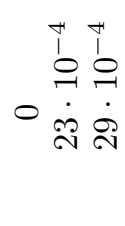 & 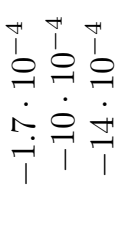 \\
\hline 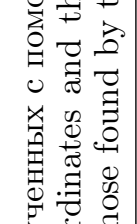 & 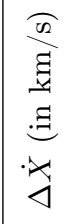 & 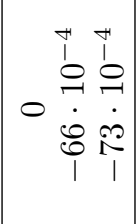 & 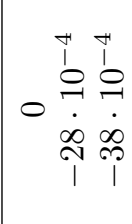 & 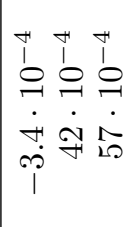 & 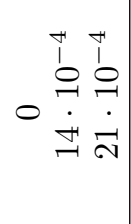 & 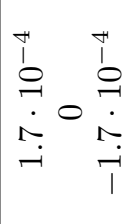 & 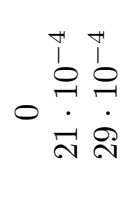 & 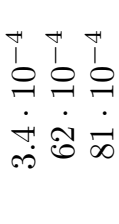 \\
\hline 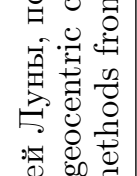 & 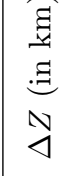 & 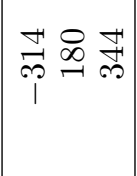 & 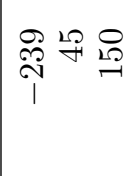 & \begin{tabular}{ccc}
0 & $m$ & $N$ \\
\hdashline & 0 & 0
\end{tabular} & @ & 年 & 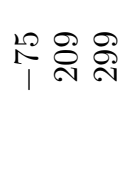 & 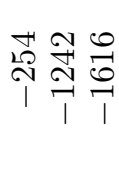 \\
\hline 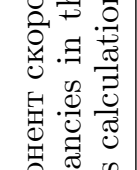 & 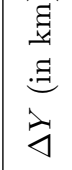 & 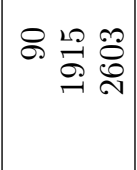 & 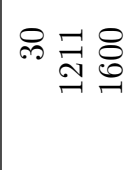 & 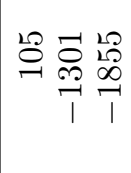 & 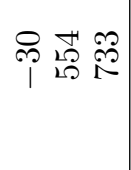 & 잉 & 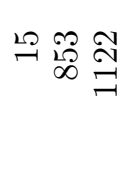 & 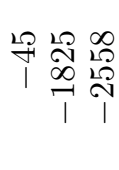 \\
\hline 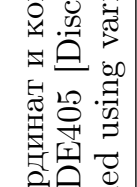 & 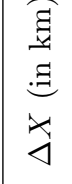 & 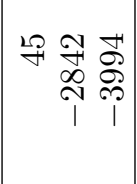 & 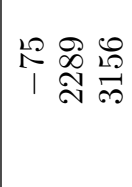 & 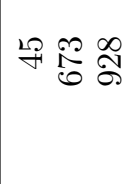 & 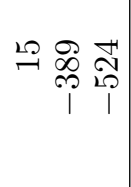 & 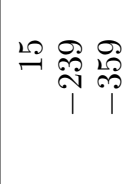 & 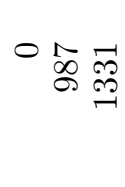 & 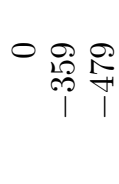 \\
\hline 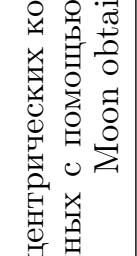 & 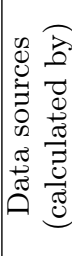 & 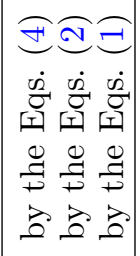 & 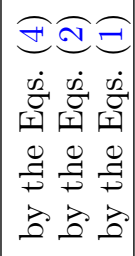 & 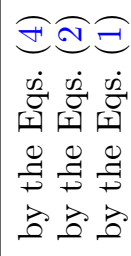 & 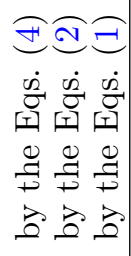 & 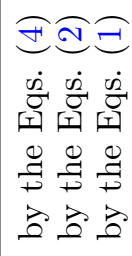 & 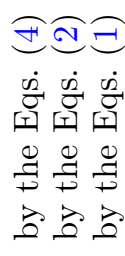 & 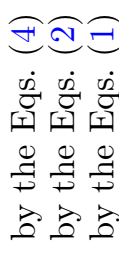 \\
\hline 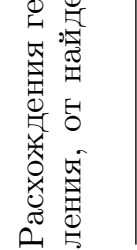 & 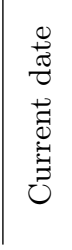 & 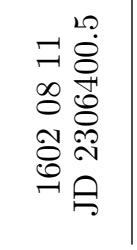 & 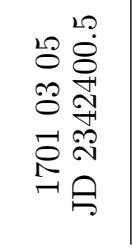 & 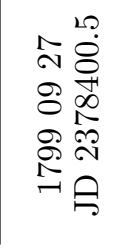 & 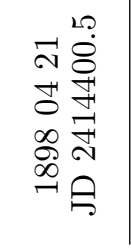 & 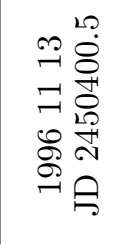 & 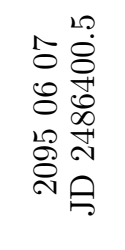 & 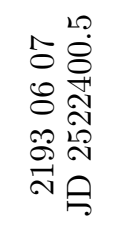 \\
\hline
\end{tabular}




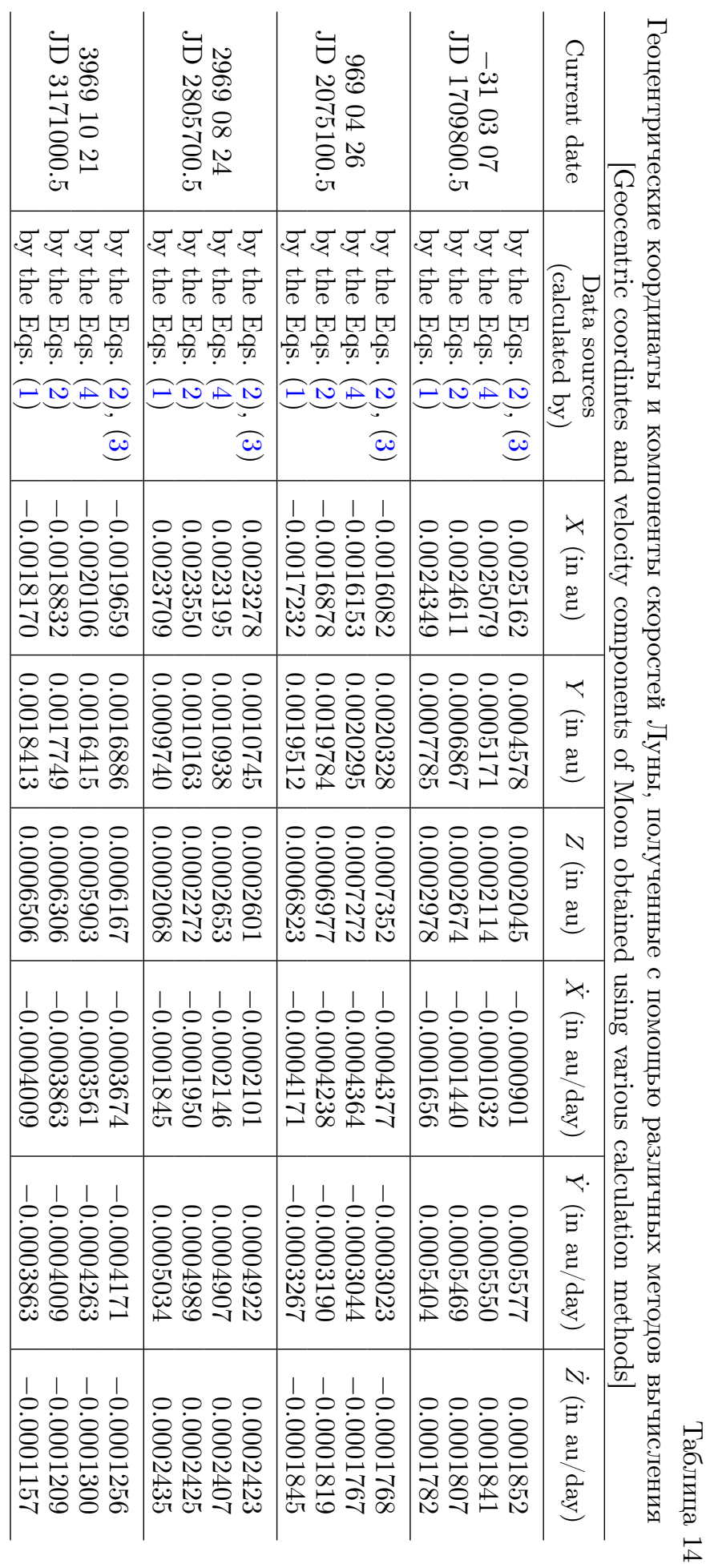




\begin{tabular}{|c|c|c|c|c|c|}
\hline 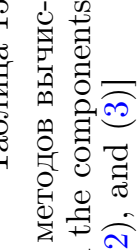 & 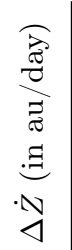 & 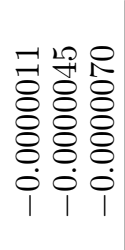 & 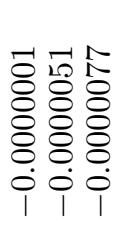 & 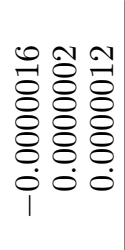 & 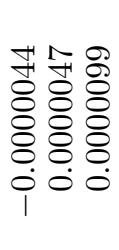 \\
\hline 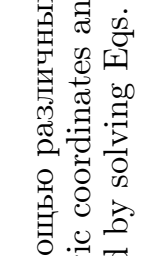 & 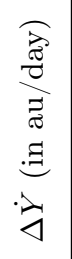 & 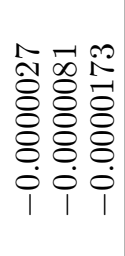 & 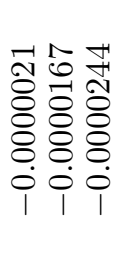 & 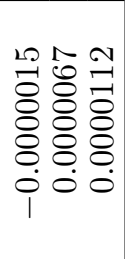 & 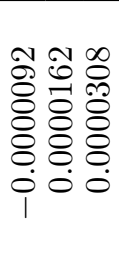 \\
\hline 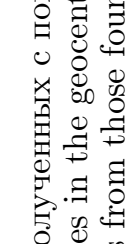 & 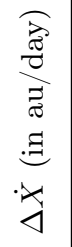 & 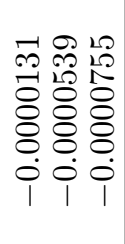 & 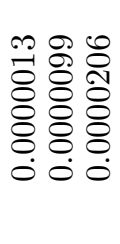 & 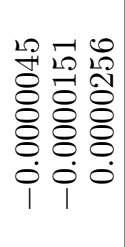 & 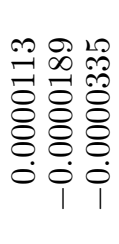 \\
\hline 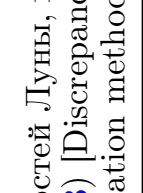 & $\begin{array}{l}\mathcal{\Xi} \\
\Xi \\
\Xi \\
y \\
y\end{array}$ & 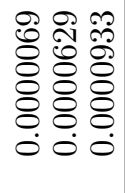 & 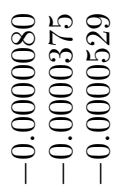 & 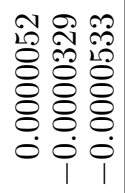 & 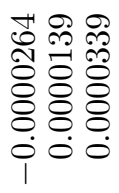 \\
\hline 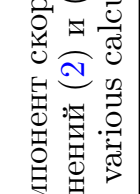 & 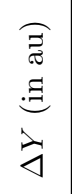 & 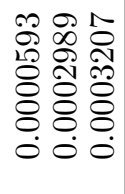 & 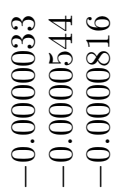 & 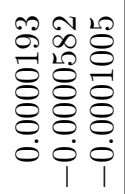 & 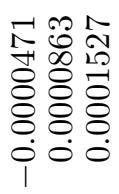 \\
\hline 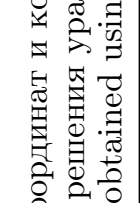 & 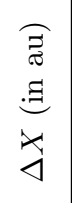 & 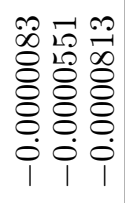 & 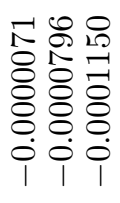 & 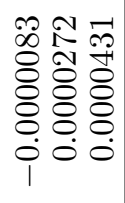 & 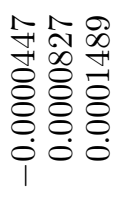 \\
\hline 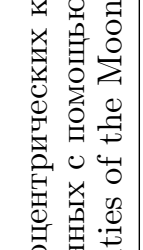 & 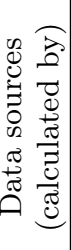 & 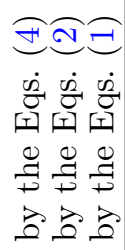 & 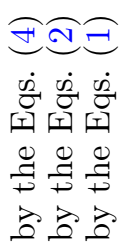 & 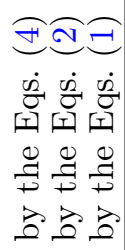 & 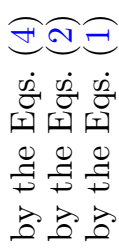 \\
\hline 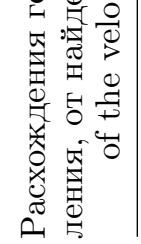 & 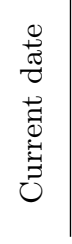 & 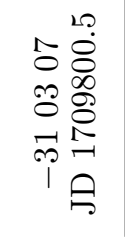 & 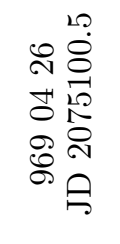 & 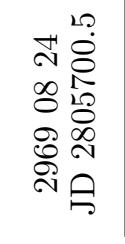 & $\infty$ \\
\hline
\end{tabular}




\begin{tabular}{|c|c|c|c|c|c|}
\hline 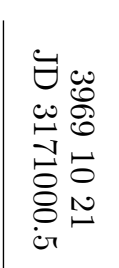 & 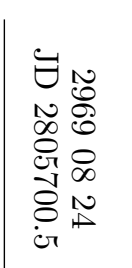 & 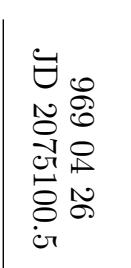 & 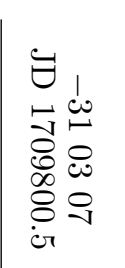 & 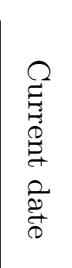 & 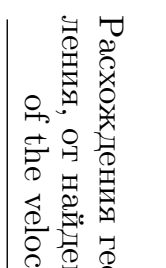 \\
\hline$\sigma \cdot \sigma \cdot \vec{\psi}$ & 将 & F्य & 将 & $\widehat{\partial}-$ & $\$ \nabla$ \\
\hline 官官宫 & 宫客官 & 官完官 & 官宽官 & 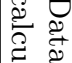 & 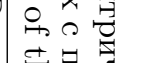 \\
\hline $\begin{array}{l}1 \\
\text { की } \\
\text { की }\end{array}$ & 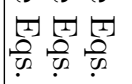 & 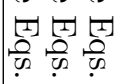 & 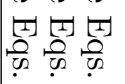 & $\begin{array}{ll}2 & \text { n } \\
0 & 0 \\
0 & 0 \\
2 & 0\end{array}$ & 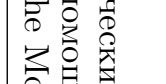 \\
\hline Ðહહ & Ðહહ & E犬E & Ðહહ & 运 & $\begin{array}{lll}0 & \ddots & x \\
0 & 0 & - \\
0 & 0 & 0\end{array}$ \\
\hline 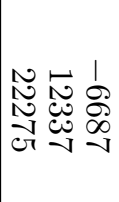 & $\begin{array}{l}\infty \\
\infty \\
\infty \\
\infty \\
\infty\end{array}$ & 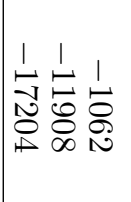 & 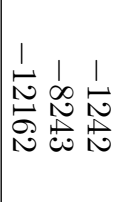 & $\begin{array}{l}D \\
\dot{X} \\
\stackrel{B}{E} \\
\frac{B}{E}\end{array}$ & 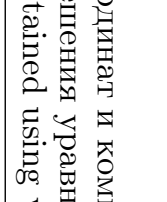 \\
\hline $\begin{array}{lll}N & 1 \\
N & 0 & 0 \\
\infty & 0 & 0 \\
1 & 0 & 0\end{array}$ & 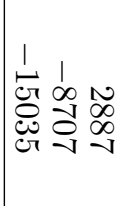 & 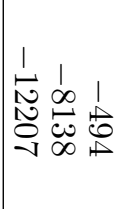 & 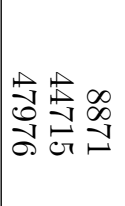 & 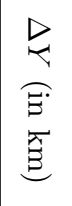 & 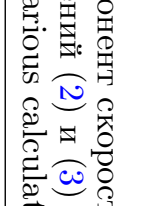 \\
\hline 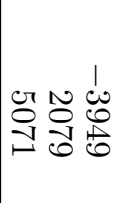 & 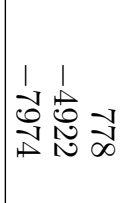 & 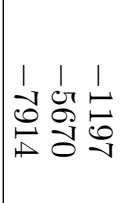 & 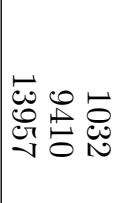 & $\begin{array}{l}D \\
\mathbb{N} \\
\stackrel{B}{E} \\
\stackrel{\bar{B}}{E}\end{array}$ & 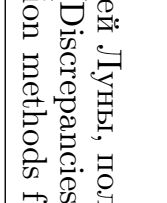 \\
\hline $\begin{array}{lll}0 & 0 & 0 \\
0 & 0 & 0 \\
ن & 0 & 0 \\
\infty & 0 & 0 \\
\infty & 0 & 0\end{array}$ & $\begin{array}{lll}0 & 1 & 1 \\
0 & 0 & 0 \\
0 & 0 & 0 \\
\mathbb{A} & 0 & 0\end{array}$ & 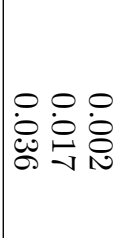 & $\mid \begin{array}{lll}1 & 1 & 1 \\
0 & 0 & 0 \\
i & 0 & 0 \\
0 & 0 & 0\end{array}$ & 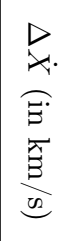 & 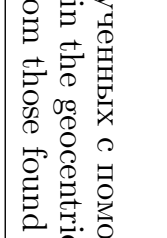 \\
\hline 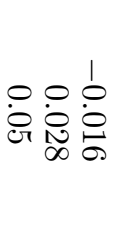 & $\begin{array}{lll}0 & 0 & 1 \\
0 & 0 & 0 \\
0 & 0 & 0 \\
0 & 0 & 0 \\
0 & N & 0\end{array}$ & $\begin{array}{lll}1 & 1 & 1 \\
0 & 0 & 0 \\
0 & 0 & 0 \\
\mathbb{N} & 0 & 0 \\
0 & \mathbb{1}\end{array}$ & $\begin{array}{lll}1 & 1 & 1 \\
0 & 0 & 0 \\
0 & 0 & 0 \\
ن & \ddots & 0\end{array}$ & 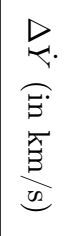 & 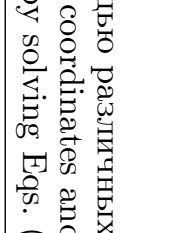 \\
\hline 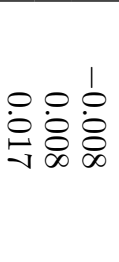 & $\begin{array}{lll}0 & 0 & 0 \\
0 & 0 & 0 \\
0 & 0 & 0 \\
0 & 8 & 0 \\
N & 0 & 0\end{array}$ & $\begin{array}{lll}1 & 1 & 1 \\
0 & 0 & 0 \\
0 & 0 & 0 \\
0 & 0 & 8 \\
ن & 0 & 0 \\
0\end{array}$ & $\begin{array}{lll}1 & 1 & 1 \\
0 & 0 & 0 \\
0 & 0 & 0 \\
0 & \wp & 0 \\
N & \infty & 0\end{array}$ & 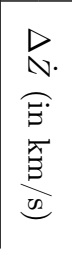 & 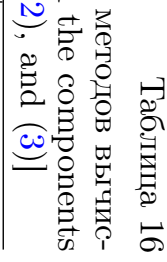 \\
\hline
\end{tabular}


ренциальных уравнений (4), в третьей и четвертой - расхождения координат, полученных с помощью решения уравнений (2) и уравнений (1).

На концах интервала интегрирования различия в координатах $X, Y$ и $Z$ на дату 7 марта 31 г. до н.э., полученных с помощью решения дифференциальных уравнений (4) и найденных с помощью совместного решения уравнений (2) и (3), составляют $\Delta X=1242$ км, $\Delta Y=-8871$ км и $\Delta Z=1032$ км соответственно. Полученные различия в геоцентрических координатах Луны, найденных с помощью совместного решения уравнений (2) и (3) и путем решения уравнений (4), являются следствием различных вековых смещений перигелиев Земли+Луны, найденных с помощью решения этих уравнений.

Расхождения геоцентрических координат Луны, найденных путем решения уравнений (2), от координат, найденных с помощью совместного решения уравнений (2) и (3) на дату 7 марта 31 г. до н.э., еще более значительны по сравнению с предыдущим случаем, т.к. по координатам $X, Y$ и $Z$ отклонения составляют минус 8243 км, 44715 км и 9410 км соответственно.

Поскольку точность геоцентрических координат Луны, найденных путем решения уравнений (4), значительно превышает точность координат Луны, найденных с помощью решения уравнений (2), это указывает на то, что решение уравнений (4) обеспечивает более высокую точность при исследовании движения больших планет и Луны по сравнению с решениями уравнений (2).

Как видно из данных, представленных в табл. 4-9, скорости вековых смещений долгот перигелиев Венеры, Земли +Луны и Марса, полученные с помощью решения уравнений (2) и найденные путем совместного решения уравнений (2) и (3), практически совпадают.

Принимая во внимание, что элементы орбит Венеры, Земли + Луны и Марса получены путем решения уравнений (4), точнее - решений уравнений (2), можно сделать следующие выводы:

а) решение релятивистских уравнений (2) не приводит к повышению точности координат и элементов орбит Венеры, Земли+Луны и Марса по сравнению с решениями уравнений (1);

б) на интервале времени порядка \pm 100 лет от начального момента интегрирования элементы орбит Венеры, Земли +Луны и Марса, найденные с помощью решения уравнений (1), (2) и (4), отличаются друг от друга в пределах погрешности оптических наблюдений;

в) с увеличением интервала интегрирования различия в координатах Венеры, Земли+Луны, Марса и Луны, найденных с помощью решения уравнений (4) и путем совместного интегрирования уравнений (2) и (3), учитывающих релятивистские эффекты и отклонение фигуры Земли от сфероида, возрастают; это указывает на ограниченные возможности применения современных математических моделей, описываемых уравнениями (1), (2) и (3), для исследования эволюции орбит Венеры, Земли, Марса и Луны на больших интервалах времени порядка 1000 и более лет;

г) в результате численного интегрирования уравнений (2) и (4) различия в элементах орбит Меркурия на интервале времени с 31 г. до н.э. по 3969 г. н.э. находятся в пределах ошибок наблюдений. 


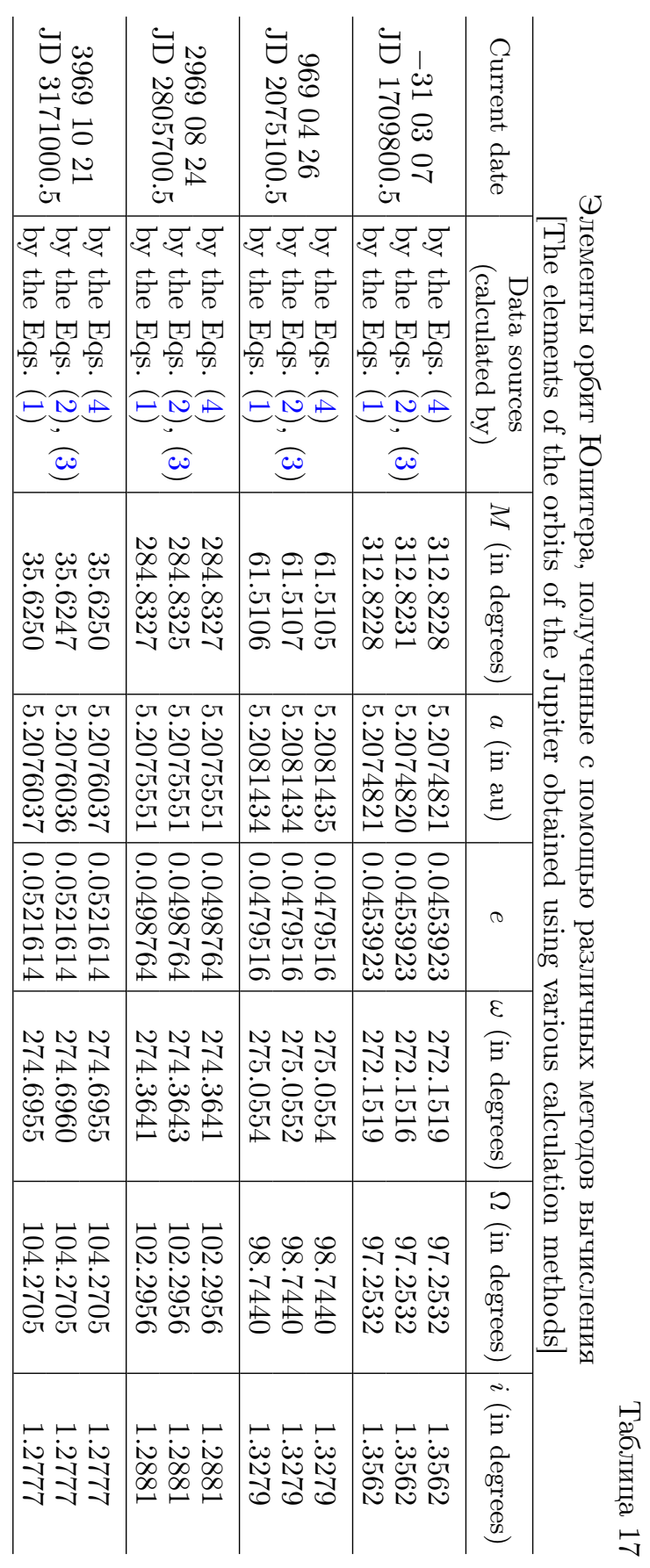




\begin{tabular}{|c|c|c|c|c|c|}
\hline \multirow{8}{*}{ 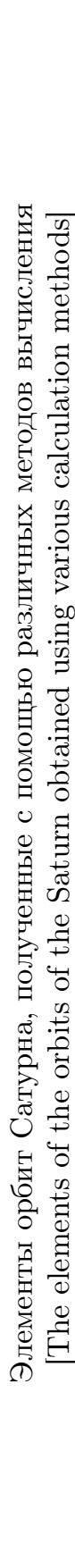 } & $\begin{array}{l}0 \\
0 \\
0 \\
0 \\
0 \\
0 \\
\Xi \\
\Xi \\
. \infty\end{array}$ & 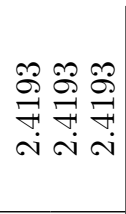 & 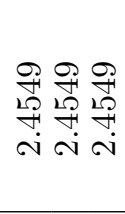 & 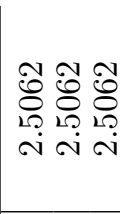 & 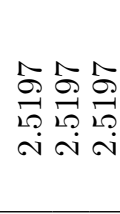 \\
\hline & 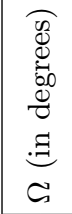 & 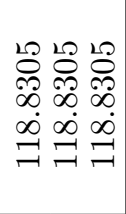 & 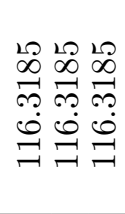 & 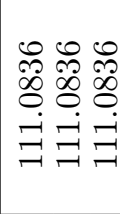 & 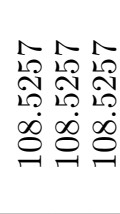 \\
\hline & $\begin{array}{l}\overparen{0} \\
0 \\
0 \\
50 \\
0 \\
0 \\
\Xi \\
3 \\
3\end{array}$ & 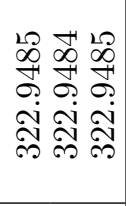 & 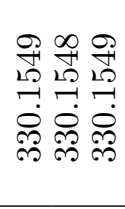 & 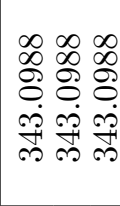 & $\begin{array}{l}8 \\
\varrho\end{array}$ \\
\hline & u & 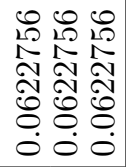 & 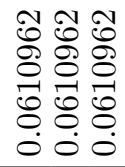 & 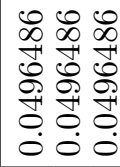 & 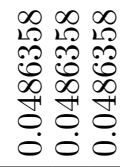 \\
\hline & $\begin{array}{l}\text { శే } \\
. \Xi \\
0 \\
0\end{array}$ & 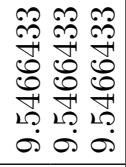 & 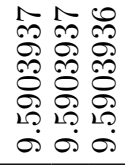 & 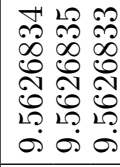 & 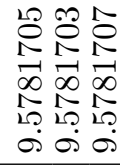 \\
\hline & 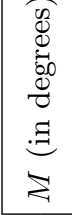 & 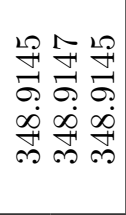 & 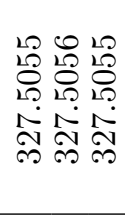 & 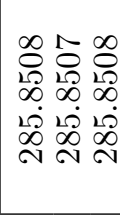 & 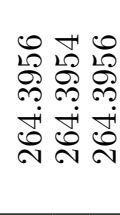 \\
\hline & 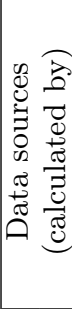 & 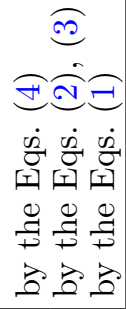 & 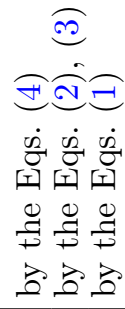 & 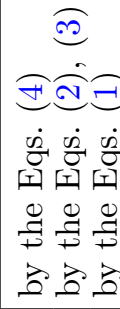 & 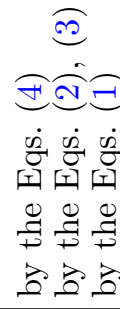 \\
\hline & 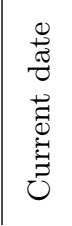 & 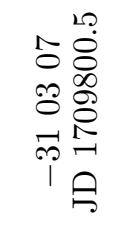 & 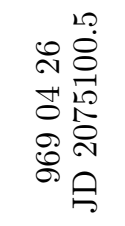 & 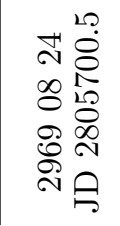 & 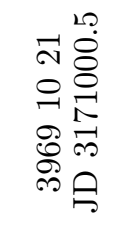 \\
\hline
\end{tabular}




\begin{tabular}{|c|c|c|c|c|c|}
\hline 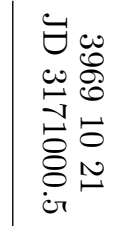 & 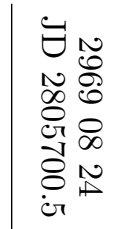 & 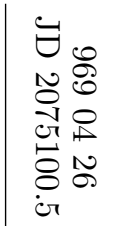 & 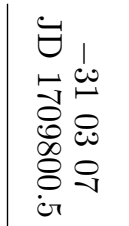 & 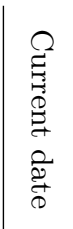 & \\
\hline$\sigma_{4} \cdot \overrightarrow{\sigma_{4}}$ & F्य $\sigma \sigma_{4}$ & F্ব & 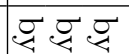 & & $\bar{G}$ \\
\hline 客客客 & 宽客完 & 客完完 & 官官客 & ఠ్ & \\
\hline 的 & 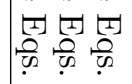 & 乐 & 至 & $\begin{array}{ll}2 & 0 \\
0 & 0 \\
0 & 0 \\
0 & 0\end{array}$ & 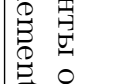 \\
\hline 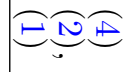 & $\overparen{E} \mathbb{E}$ & $Ð \mathbb{E}$ & 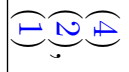 & 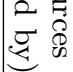 & 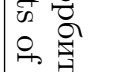 \\
\hline 厄િ & త્త & త્ల & త્త & & 帘 \\
\hline $\begin{array}{l}0.00 \\
0 \\
0\end{array}$ & 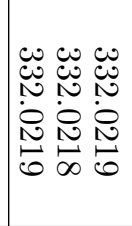 & 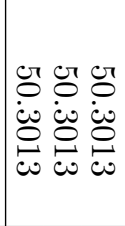 & 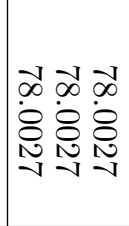 & 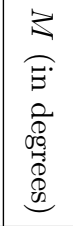 & 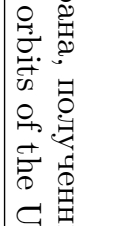 \\
\hline 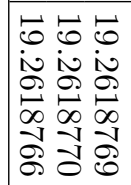 & 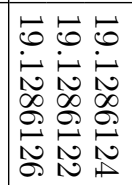 & 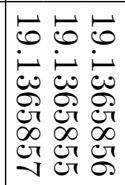 & 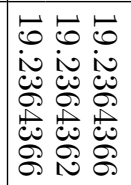 & $\begin{array}{l}\stackrel{2}{\Xi} \\
\stackrel{\Xi}{E}\end{array}$ & 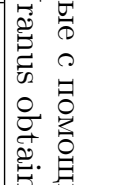 \\
\hline 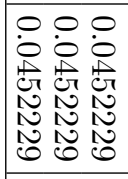 & 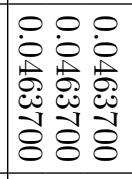 & 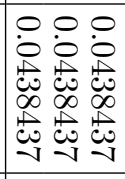 & 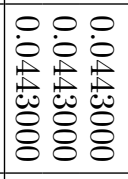 & C & 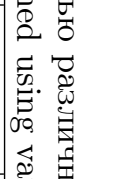 \\
\hline نِ & 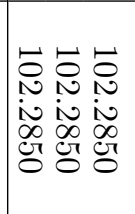 & 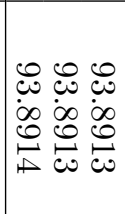 & 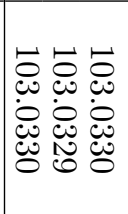 & 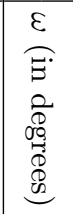 & 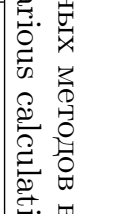 \\
\hline 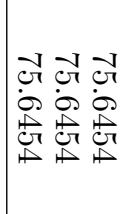 & 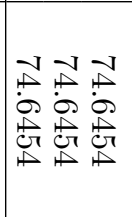 & 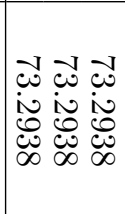 & 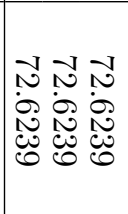 & 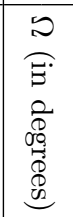 & 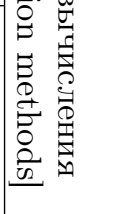 \\
\hline 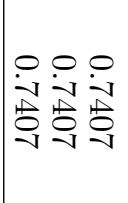 & 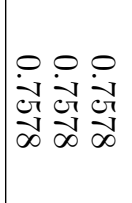 & $\mid \begin{array}{lll}0 & 0 & 0 \\
\ddots & \ddots & \ddots \\
\infty & \infty & 0 \\
0 & \infty & 0 \\
0 & 0 & 0 \\
0 & 0 & 0\end{array}$ & $\mid \begin{array}{ccc}0 & 0 & 0 \\
\infty & \infty & \infty \\
0 & 0 & 0 \\
\infty & 0 & \wp \\
0 & 0 & 0\end{array}$ & 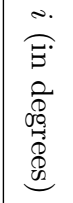 & \\
\hline
\end{tabular}




\begin{tabular}{|c|c|c|c|c|c|}
\hline \multirow{8}{*}{ 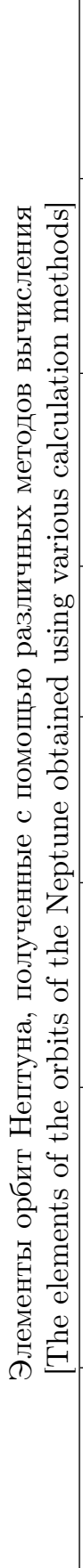 } & 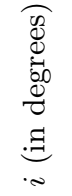 & 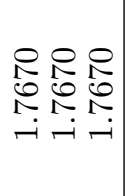 & $\begin{array}{l}N N N \\
0 N 0 \\
N N N \\
-1\end{array}$ & 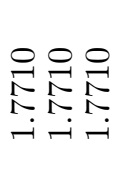 & 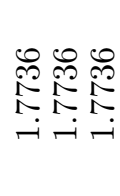 \\
\hline & 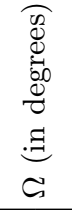 & 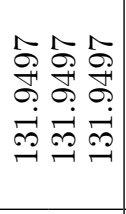 & 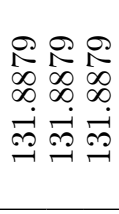 & 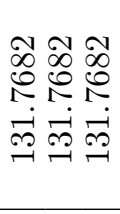 & 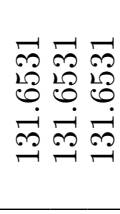 \\
\hline & $\begin{array}{l}\overparen{0} \\
0 \\
0 \\
0 \\
0 \\
0 \\
\Xi \\
\Xi \\
3\end{array}$ & 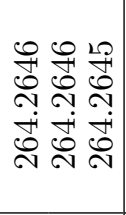 & 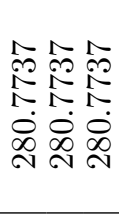 & 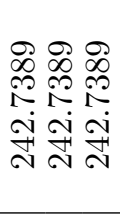 & 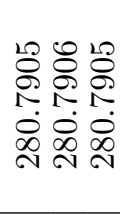 \\
\hline & 0 & 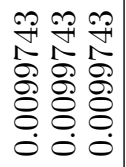 & 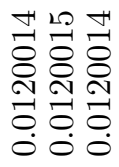 & $\begin{array}{lll}0 & 0 \\
0 & 0 \\
1 & 0 \\
0 & 0 & 0 \\
0 & 0 & 1 \\
0 & 0 & 0 \\
0 & 0 & 0 \\
0 & 0 & 0\end{array}$ & 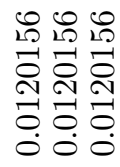 \\
\hline & $\begin{array}{l}\text { Fే } \\
\Xi \\
\Xi \\
0\end{array}$ & 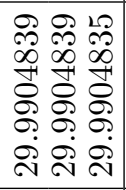 & 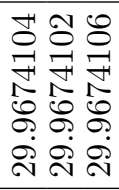 & 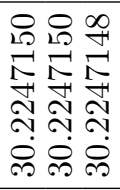 & 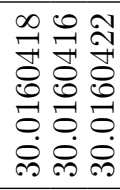 \\
\hline & $\begin{array}{l}0 \\
0 \\
0 \\
0 \\
0 \\
0 \\
0 \\
\Xi \\
\Xi \\
z\end{array}$ & 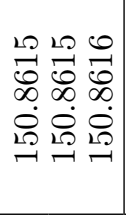 & 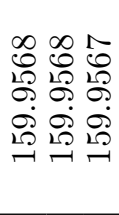 & 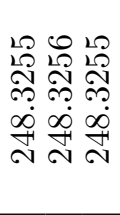 & 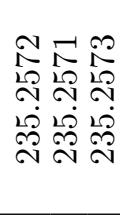 \\
\hline & 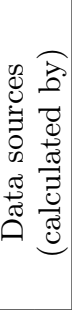 & 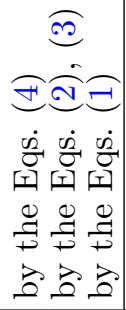 & 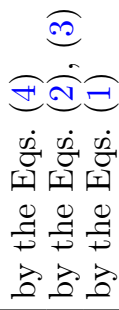 & 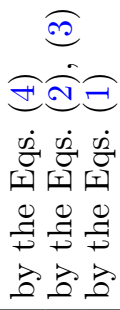 & 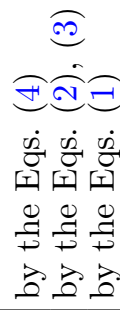 \\
\hline & 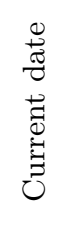 & 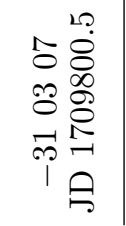 & 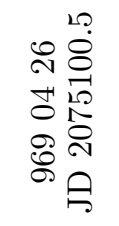 & 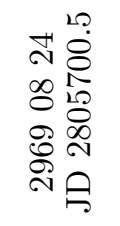 & 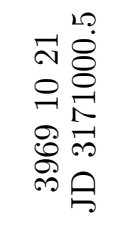 \\
\hline
\end{tabular}




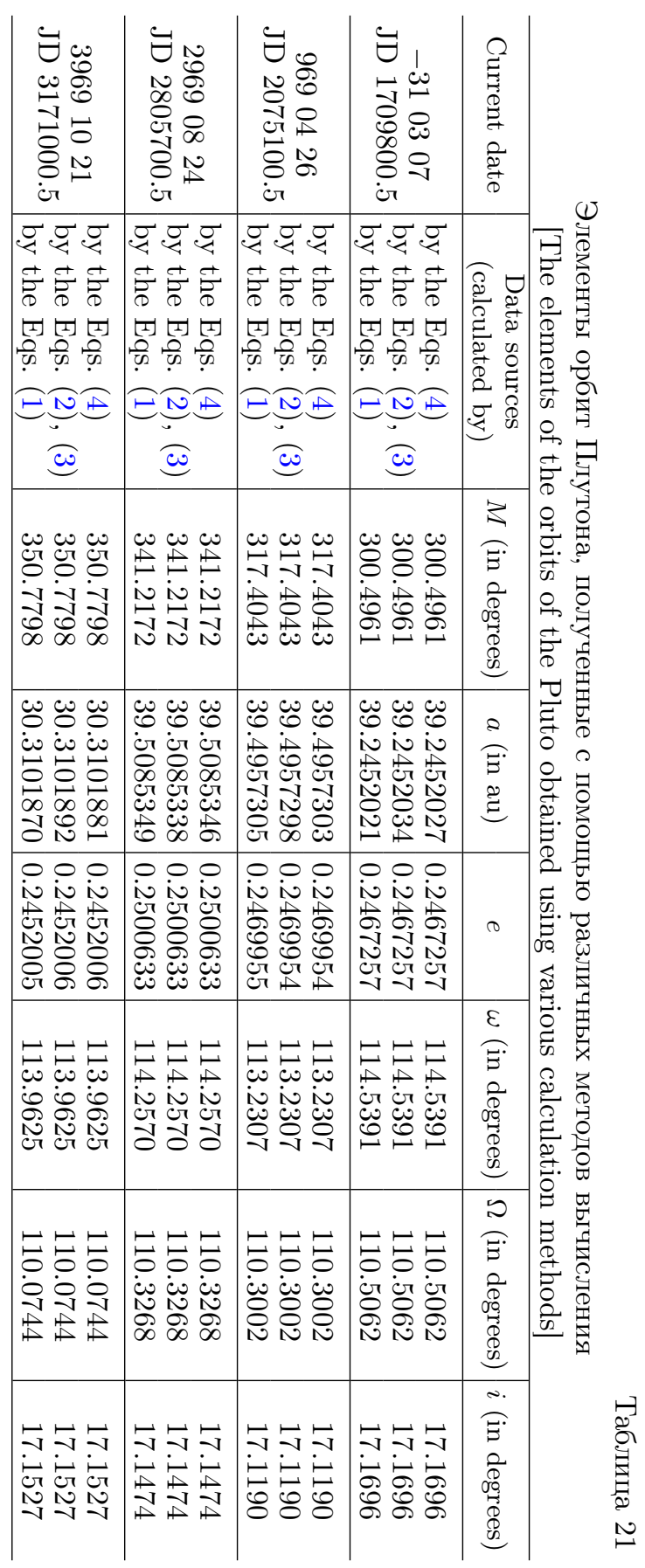


Несмотря на ограниченные возможности применения дифференциальных уравнений движения (1) и (2) к вышеуказанным объектам, данные уравнения применимы к исследованию эволюции орбит внешних планет ЮпитерПлутон на интервале времени 4000 лет. В табл. 17-21 представлены элементы орбит Юпитера-Плутона на интервале времени с 7 марта 31 г. до н.э. по 21 октября 3969 г. н.э. Как показывают результаты вычислений (см. табл. 17-21), для исследования эволюции орбит данных объектов на интервале времени порядка 1000 лет можно использовать наряду с уравнениями (4) уравнения (1) и (2).

В отличие от ньютоновых и релятивистских уравнений, решение уравнений (4) позволяет получить координаты больших планет и Луны на интервале 600 лет (1600-2200 гг.), полностью согласованные с наблюдениями без привлечения дополнительных уравнений.

В заключение следует отметить, что дифференциальные уравнения (4) свободны от недостатков, свойственных уравнениям (2), т.к. их использование для решения рассмотренных ранее задач приводит к сокращению рабочего времени программы численного интегрирования и повышению точности конечных результатов более чем на порядок. Кроме того, они могут быть эффективно использованы для исследования эволюции орбит больших планет и Луны на длительных интервалах времени порядка 1000 и более лет.

Конкурирующие интересы. Заявляем, что в отношении авторства и публикации этой статьи конфликта интересов не имеем.

Авторский вклад и ответственность. Все авторы принимали участие в разработке концепции статьи и в написании рукописи. Авторы несут полную ответственность за предоставление окончательной рукописи в печать. Окончательная версия рукописи была одобрена всеми авторами.

Финансирование. Исследование выполнялось без финансирования.

\section{Библиографический список}

1. Чеботарев Г. А. Аналитические и численные методы небесной механики. М., Л.: Наука, 1965. 368 с.

2. Субботин М. Ф. Введение в теоретическую астрономию. М.: Наука, 1968. 800 с.

3. Newhall X. X., Standish E M., Williams J. G. DE 102: A numerically integrated ephemeris of the Moon and planets spanning forty-four centuries // Astron. Astrophys., 1983. vol. 125, no. 1. pp. 150-167.

4. Заусаев А. Ф., Заусаев А. А. Математическое моделирование орбитальной эволючии малых тел Солнечной системы. М.: Машиностроение-1, 2008. 250 с.

5. Заусаев А. Ф. Исследование движения планет, Луны и Солнца, основанное на новом принципе взаимодействия // Вестн. Сам. гос. техн. ун-та. Сер. Физ.-мат. науки, 2014. №3(36). С. 118-131. doi: 10.14498/vsgtu1304.

6. Заусаев А. Ф. Сопоставление координат больших планет, Луны и Солнца, полученных на основе нового принципа взаимодействия и банка данных DE405// Becmн. Сам. гос. техн. ун-та. Сер. Физ.-мат. науки, 2016. Т. 20, №1. С. 121-148. doi: 10.14498/ vsgtu1458.

7. Заусаев А. Ф., Романюк М. А. Численнъе методы в задачах математического моделирования двиюения небесных тел в Солнечной системе. Самара: СамГТУ, 2017. $265 \mathrm{c}$.

8. Красинский Г. А., Питьева Е. В., Свешников М. Л., Свешникова Е. С. Уточнение эфемерид внутренних планет и Луны по радиолокационным, лазерным и мередианным измерениям 1961-1980 гг. // Бюлл. ИТА АН СССР, 1982. Т. 15, № 3. С. 145-163. 
9. Заусаев А. Ф., Заусаев А. А, Ольхин А. Г. Численное интегрирование уравнений движения больших планет (Меркурий-Плутон) и Луны с учетом радиолокационных наблюдений // Вестн. Сам. гос. техн. ун-та. Сер. Физ.-мат. науки, 2004. № 26. С. 43-47. doi: $10.14498 /$ vsgtu175.

10. Заусаев А. Ф. Теория движения $n$ материальных тел, основанная на новом принципе взаимодействия // Вестн. Сам. гос. техн. ун-та. Сер. Физ.-мат. науки, 2006. № 43. C. $132-139$. doi: $10.14498 /$ vsgtu463.

11. Standish E. M. JPL Planetary and Lunar Ephemerides: DE405/LE405. Interoffice memorandum: JPL IOM 312. F-98-048, 1998, August 26. 18 pp., ftp://ssd.jpl.nasa.gov/pub/ eph/planets/ioms/de405.iom.pdf.

12. Питьева Е. В. Современные численные теории движения Солнца, Луны и больших планет / Эфемеридная астрономия/ Труды ИПА РАН, Т. 10. М.: Ин-т приклад. астрономии. С. 112-134.

13. Питьева Е. В. Высокоточные эфемериды планет - ЕРМ и определение некоторых астрономических постоянных // Астрономический вестник, 2005. Т. 39, № 3. С. 202-213.

14. Pitjeva E. V., Bratseva O. A., Panfilov V. E. EPM - Ephemerides of Planets and the Moon of IAA RAS: Their model, accuracy, availability / Proc. of the Journées 2010 "Systèmes de Référence Spatio-Temporels" (JSR2010): New challenges for reference systems and numerical standards in astronomy (Observatoire de Paris, 20-22 September 2010); ed. N. Capitaine, 2010. pp. 49-54.

15. Pitjeva E. V., Pitjev N. P. Development of planetary ephemerides EPM and their applications // Celest. Mech. Dyn. Astr., 2014. vol.119, no.3. pp. 237-256. doi:10.1007/ s10569-014-9569-0.

16. Simon J.-L., Francou G., Fienga A., Manche H. New analytical planetary theories VSOP2013 and TOP2013// Astron. Astrophys., 2013. vol.557, A49. doi: 10.1051/ 0004-6361/201321843.

17. Folkner W. M., Williams J. G., Boggs D. H., Park R. S., Kuchynka P. The Planetary and Lunar Ephemerides DE430 and DE431: IPN Progress Report, 42-196, 2014, February 15. 81 pp., https://ipnpr.jpl.nasa.gov/progress_report/42-196/196C.pdf.

18. Everhart E. Implicit single-sequence methods for integrating orbits // Celestial Mech., 1974. vol. 10, no. 1. pp. 35-55. doi : 10.1007/BF01261877.

19. Визгин В. П. Релятивистская теория тяготения (истоки и формирование, 1900-1915). М.: Наука, 1981. 352 с.

20. Le Verrier U. J. Theorie du movement de Mercure / Annales de l'Observatoire imperial de Paris. vol. 5: Annales de l'Observatoire de Paris. Memoires. Paris: Mallet-Bachelier, 1859. 195 pp.

21. Roseveare N. T. Mercury's perihelion from Le Verrier to Einstein. Oxford: Clarendon Press, 1982. viii +208 pp.

22. Богородский А. Ф. Всемирное тяготение. Киев: Наукова думка, 1971. 352 с.

23. Брумберг В. А. Релятивистская небесная механика. М.: Наука, 1972. 384 с.

24. Кислик М. Д., Колюка Ю. Ф., Котельников В. А., Петров Г.М., Тихонов В. Ф. Единая релятивистская теория движения внутренних планет Солнечной системы // Докл. АН СССР, 1980. Т. 255, № 3. С. 545-547; Кислик М. Д., Колюка Ю. Ф., Котельников В. А., Петров Г. М., Тихонов В. Ф. Единая релятивистская теория движения внутренних планет Солнечной системы. Релятивистские эффекты при определении орбит планет по радиолокационным наблюдениям, Научная сессия Отделения общей физики и астрономии и Отделения ядерной физики Академии наук СССР (26-27 ноября 1980 г.) // УФН, 1981. T. 134, № 1. С. 165-166. doi : 10.3367/UFNr.0134.198105j.0165.

25. Кислик М. Д. Релятивистские эффекты при определении орбит планет по радиолокационным наблюдениям // Писъма в Астрономический журнал, 1981. Т. 7, № 1. С. 56-60. 
MSC: 70F15, 70M20, 65L05

\title{
Comparison of various mathematical models on the example of solving the equations of the movement of large planets and the Moon
}

\author{
A. F. Zausaev, M. A. Romanyuk \\ Samara State Technical University, \\ 244, Molodogvardeyskaya st., Samara, 443100, Russian Federation.
}

\begin{abstract}
In this paper, we study the accuracy of solving various differential equations describing the motion of large planets, the Moon and Sun. On the time interval from 31 years $\mathrm{BC}$ to $3969 \mathrm{AD}$, the numerical integration of Newtonian, relativistic differential equations, and equations obtained on the basis of the interaction of the surrounding space with moving material bodies was carried out. The range of applicability of the considered differential equations for the investigated objects is revealed. By comparing of the coordinates of the Moon, found by solving various differential equations and the DE405 data bank, it is shown that the greatest accuracy in the elements of the orbits of large planets is achieved by solving differential equations obtained on the basis of the interaction of the surrounding space with moving material bodies. The solution of relativistic equations provides high accuracy of the orbit elements for Mercury and the outer planets throughout the integration interval. However, for the remaining inner planets and the Moon, the accuracy of the orbital elements obtained by solving relativistic equations is comparable to the accuracy obtained by solving Newton equations. It is believed that the use of the harmonic coordinate system is justified only for Mercury from the point of view of the velocity of the secular longitude displacement of its perihelion, but for other internal planets (the Venus, Earth \& Moon, and Mars) the velocities of secular displacements of the longitude of the perihelion's are overstated. It is shown that the solution of differential equations obtained on the basis of the interaction of the surrounding space with moving material bodies ensures a high accuracy of obtaining orbital elements for all objects under consideration on the time interval under study.
\end{abstract}

\section{Research Article}

(우 (i) The content is published under the terms of the Creative Commons Attribution 4.0 International License (http://creativecommons.org/licenses/by/4.0/)

Please cite this article in press as:

Zausaev A. F., Romanyuk M. A. Comparison of various mathematical models on the example of solving the equations of the movement of large planets and the Moon, Vestn. Samar. Gos. Tekhn. Univ., Ser. Fiz.-Mat. Nauki [J. Samara State Tech. Univ., Ser. Phys. Math. Sci.], 2019, vol. 23, no. 1, pp. 152-185. doi: 10.14498/vsgtu1663 (In Russian).

Authors' Details:

Anatoliy F. Zausaev (10) https://orcid.org/0000-0002-5035-9615

Dr. Phys. \& Math. Sci.; Professor; Dept. of Applied Mathematics \& Computer Science; e-mail: zausaev_af@mail.ru

Mariya A. Romanyuk (D) https://orcid.org/0000-0003-0796-2061

Cand. Tech. Sci.; Associate Professor; Dept. of Applied Mathematics \& Computer Science; e-mail: zausmasha@mail.ru 
Keywords: orbital elements, numerical integration, differential equation of motion.

Received: $6^{\text {th }}$ December, 2018 / Revised: $27^{\text {th }}$ February, 2019/

Accepted: $4^{\text {th }}$ March, 2019 / First online: $15^{\text {th }}$ March, 2019

Competing interests. We declare that we have no conflicts of interest in the authorship and publication of this article.

Authors' contributions and responsibilities. Each author has participated in the article concept development and in the manuscript writing. The authors are absolutely responsible for submitting the final manuscript in print. Each author has approved the final version of manuscript.

Funding. This research received no specific grant from any funding agency in the public, commercial, or not-for-profit sectors.

\section{References}

1. Chebotarev G. A. Analytical and Numerical Methods of Celestial Mechanics, Modern Analytic and Computational Methods in Science and Mathematics, vol. 9, American Elsevier Publishing Co., Inc., 1967, xviii+331 pp.

2. Subbotin M. F. Vvedenie v teoreticheskuiu astronomiiu [Introduction to theoretical astronomy]. Moscow, Nauka, 1968, 800 pp. (In Russian)

3. Newhall X. X., Standish E M., Williams J. G. DE 102: A numerically integrated ephemeris of the Moon and planets spanning forty-four centuries, Astron. Astrophys., 1983, vol. 125, no. 1 , pp. 150-167.

4. Zausaev A. F., Zausaev A. A. Matematicheskoe modelirovanie orbital'noi evoliutsii malykh tel Solnechnoi sistemy [Mathematical modelling of orbital evolution of small bodies of the Solar system]. Moscow, Mashinostroenie-1, 2008, 250 pp. (In Russian)

5. Zausaev A. F. The Investigation of the Motion of Planets, the Moon, and the Sun Based on a New Principle of Interaction, Vestn. Samar. Gos. Tekhn. Univ., Ser. Fiz.-Mat. Nauki [J. Samara State Tech. Univ., Ser. Phys. Math. Sci.], 2014, no. 3(36), pp. 118-131 (In Russian). doi : $10.14498 /$ vsgtu1304.

6. Zausaev A. F. Comparison of the coordinates of the major planets, the Moon, and the Sun obtained based on a new principle of interaction and of the data bank DE405, Vestn. Samar. Gos. Tekhn. Univ., Ser. Fiz.-Mat. Nauki [J. Samara State Tech. Univ., Ser. Phys. Math. Sci.], 2016, vol. 20, no. 1, pp. 121-148 (In Russian). doi: 10.14498/vsgtu1458.

7. Zausaev A. F., Romaniuk M. A. Chislennye metody $v$ zadachakh matematicheskogo modelirovaniia dvizheniia nebesnykh tel $v$ Solnechnoi sisteme [Numerical methods in problems of mathematical modeling of motion of celestial bodies in the Solar system]. Samara, Samara State Technical Univ., 2017, 265 pp. (In Russian)

8. Krasinskii G. A., Piteva E. V., Sveshnikov M. L., Sveshnikova E. S. Improvement of the ephemerides of the inner planets and the moon using radar, laser, and meridian measurements during 1961-1980, Institut Teoreticheskoi Astronomii, Biulleten, 1982, vol. 15, no. 3, pp. 145-163 (In Russian).

9. Zausaev A. F., Zausaev A. A., Ol'khin A. G. The numerical integration of the equations of motion for large planets (Mercury and Pluto) and Moon with the radar observations, Vestn. Samar. Gos. Tekhn. Univ., Ser. Fiz.-Mat. Nauki [J. Samara State Tech. Univ., Ser. Phys. Math. Sci.], 2004, no. 26, pp. 43-47 (In Russian). doi: 10.14498/vsgtu175.

10. Zausaev A. F. Theory of motion of $n$ material bodies, based on a new interaction principle, Vestn. Samar. Gos. Tekhn. Univ., Ser. Fiz.-Mat. Nauki [J. Samara State Tech. Univ., Ser. Phys. Math. Sci.], 2006, no. 43, pp. 132-139 (In Russian). doi: 10.14498/vsgtu463. 
11. Standish E. M. JPL Planetary and Lunar Ephemerides, DE405/LE405. Interoffice memorandum: JPL IOM 312. F-98-048, 1998, August 26, 18 pp., ftp://ssd.jpl.nasa.gov/pub/ eph/planets/ioms/de405.iom.pdf.

12. Pitjeva E. V. Modern numerical ephemerides of the Sun, the Moon and majorplanets, In: Efemeridnaia astronomiia [Ephemeris Astronomy], Proc. of IPA RAS, 10. Moscow, IPA RAS, pp. 112-134 (In Russian).

13. Pitjeva E. V. High-precision ephemerides of planets-EPM and determination of some astronomical constants, Solar System Research, 2005, vol.39, no.3, pp. 176-186. doi: 10.1007/ s11208-005-0033-2.

14. Pitjeva E. V., Bratseva O. A., Panfilov V. E. EPM - Ephemerides of Planets and the Moon of IAA RAS: Their model, accuracy, availability, In: Proc. of the Journées 2010 "Systèmes de Référence Spatio-Temporels" (JSR2010): New challenges for reference systems and numerical standards in astronomy (Observatoire de Paris, 20-22 September 2010); ed. N. Capitaine, 2010, pp. 49-54.

15. Pitjeva E. V., Pitjev N. P. Development of planetary ephemerides EPM and their applications, Celest. Mech. Dyn. Astr., 2014, vol.119, no.3, pp. 237-256. doi:10.1007/ s10569-014-9569-0.

16. Simon J.-L., Francou G., Fienga A., Manche H. New analytical planetary theories VSOP2013 and TOP2013, Astron. Astrophys., 2013, vol.557, A49. doi: 10.1051/ 0004-6361/201321843.

17. Folkner W. M., Williams J. G., Boggs D. H., Park R. S., Kuchynka P. The Planetary and Lunar Ephemerides DE430 and DE431, IPN Progress Report, 42-196, 2014, February 15, 81 pp., https://ipnpr.jpl.nasa.gov/progress_report/42-196/196C.pdf.

18. Everhart E. Implicit single-sequence methods for integrating orbits, Celestial Mech., 1974, vol. 10, no. 1, pp. 35-55. doi: 10.1007/BF01261877.

19. Vizgin V. P. Reliativistskaia teoriia tiagoteniia (istoki i formirovanie, 1900-1915) [The relativistic theory of gravitation. Sources and formation, 1900-1915]. Moscow, Nauka, 1981, 352 pp. (In Russian)

20. Le Verrier U. J. Theorie du movement de Mercure, Annales de l'Observatoire imperial de Paris, vol. 5, Annales de l'Observatoire de Paris. Memoires. Paris, Mallet-Bachelier, 1859, 195 pp.

21. Roseveare N. T. Mercury's perihelion from Le Verrier to Einstein. Oxford, Clarendon Press, 1982, viii +208 pp.

22. Bogorodsky A. F. Vsemirnoe tiagotenie [Universal Gravitation]. Kiev, Naukova Dumka, 1971, 352 pp. (In Russian)

23. Brumberg V. A. Reliativistskaia nebesnaia mekhanika [Relativistic Celestial Mechanics]. Moscow, Nauka, 1972, 384 pp. (In Russian)

24. A Unified Relativistic Theory of the Motion of the Inner Planets of the Solar System, Proceedings of the USSR Academy of Sciences, 1980, T. 255, № 3, C. 545-547 (In Russian); Kislik M. D., Kolyuka Yu. F., Kotel'nikov V. A., Tikhonov V. F. A Unified Relativistic Theory of the Motion of the Inner Planets of the Solar System. Relativistic Effects in Determination of the Orbits of the Planets from Radar Observations, Sov. Phys. Usp., 1981, vol. 24, no. 1, pp. 437-438. doi : 10.1070/PU1981v024n05ABEH004807.

25. Kislik M. D. Relativistic effects in radar determinations of planetary orbits, Soviet Astronomy Letters, 1981, vol. 7, no. 1, pp. 31-34. 